\title{
Dimensional reduction of M5 branes
}

\author{
Andreas Gustavsson* \\ Physics Department, University of Seoul, Seoul 02504, Korea
}

(Received 14 November 2021; accepted 1 February 2022; published 15 February 2022)

\begin{abstract}
We study dimensional reduction of M5 branes on a circle bundle when the supersymmetry parameter is not constant along the circle. When the gauge group is Abelian and the fields appear quadratically in the Lagrangian, we can always obtain a supersymmetric five-dimensional theory by keeping fermionic nonzero modes that match with the corresponding nonzero modes of the supersymmetry parameter, and by keeping the zero modes for the bosonic fields as usual. But a supersymmetric non-Abelian generalization can be found only under special circumstances. One instance where we find a non-Abelian supersymmetric generalization is when we perform dimensional reduction along a null direction.
\end{abstract}

DOI: 10.1103/PhysRevD.105.046010

\section{INTRODUCTION}

There is a supersymmetric $(2,0)$ Abelian tensor multiplet in $\mathbb{R}^{1,5}$ which has a self-dual three-form, five scalar fields and four real Weyl fermionic fields. We can put this tensor multiplet on any six-manifold for which there exists a nontrivial solution to the six-dimensional conformal Killing spinor equation

$$
\nabla_{M} \varepsilon=\Gamma_{M} \eta
$$

Here $M=0,1,2,3,4,5$ is a vector index on the sixmanifold that we will take to be Lorentzian, and $\varepsilon$ will then be the supersymmetry parameter. Equation (1.1) can be relaxed by turning on supergravity background fields. But we will not study such a generalization here. So $\nabla_{M}$ here is denoting a curvature covariant derivative that only involves the spin connection and no R-gauge field is turned on.

The classical non-Abelian tensor multiplet is not known and perhaps it does not exist. One approach is then to consider the Abelian tensor multiplet on a circle bundle and perform dimensional reduction along the circle. Then one finds an Abelian 5d Yang-Mills theory for which one can find a non-Abelian generalization. If the supersymmetry parameter is constant along the circle, then it will survive as a supersymmetry under dimensional reduction. Otherwise the supersymmetry will be broken but one may then get a supersymmetric theory by turning on a background R-gauge field that will relax the requirement (1.1). But

\footnotetext{
*agbrev@gmail.com
}

Published by the American Physical Society under the terms of the Creative Commons Attribution 4.0 International license. Further distribution of this work must maintain attribution to the author(s) and the published article's title, journal citation, and DOI. Funded by SCOAP ${ }^{3}$. that changes the problem that one may want to study. So we would like to analyze whether one can avoid turning on the R-gauge field and somehow take advantage of the fact that the $6 \mathrm{~d}$ theory is supersymmetric.

One example that one may want to study is the M5 brane on $S^{6}$ that one may conformally map to $S^{1} \times H_{5}$. If one wants to study this problem without any background fields turned on, then one finds that the supersymmetry parameter will have a nontrivial dependence on $S^{1}$ in $S^{1} \times H_{5}$, and dimensional reduction down to $H_{5}$ yields a nonsupersymmetric Yang-Mills theory that is quite difficult to study. Being a nonrenormalizable theory it has no clear welldefined perturbative expansion and there are not many tools to study this theory and supersymmetric localization cannot be used if the Yang-Mills theory one gets on $\mathrm{H}_{5}$ is not supersymmetric.

In this paper we will study the following situation. We assume that the $6 \mathrm{~d}$ theory is supersymmetric on a circle bundle with fiber coordinate $u$. We also assume that the supersymmetry parameter is not constant along $u$. So under dimensional reduction along $u$ all supersymmetry is gone. That is the case if we consider the bosonic and fermionic zero modes. But what if we consider the bosonic zero modes and some fermionic nonzero modes? Is there a consistent trunctation of supersymmetry where bosonic zero modes are kept such that supersymmetry exists in that $5 \mathrm{~d}$ truncation?

If the fields appear only quadratically in the Lagrangian so that the gauge group is Abelian, then there always exists such a consistent truncation. To see this, let us schematically write the $6 \mathrm{~d}$ Lagrangian as

$$
\mathcal{L}_{6 d}=(\partial \phi)^{2}+\psi \partial \psi
$$

where $\phi$ denotes bosonic fields and $\psi$ denotes fermionic fields. The supersymmetry variation is schematically on the form 


$$
\delta \phi=\varepsilon \psi, \quad \delta \psi=\varepsilon \partial \phi .
$$

Then the supersymmetry variation of the Lagrangian is a sum of terms on the form

$$
0=\delta \mathcal{L}_{6 d}=\sum \partial^{2} \phi \varepsilon \psi
$$

and the sum vanishes since the 6d Lagrangian is supersymmetric. Now let us make the truncation where we keep the bosonic zero mode along the $u$ direction,

$$
\phi_{0}=\int d u \phi
$$

Its supersymmetry variation is

$$
\delta \phi_{0}=\int d u \varepsilon \psi .
$$

Now let us assume that the supersymmetry parameter has only two nonzero modes,

$$
\varepsilon=e^{i a u} \varepsilon_{+1}+e^{-i a u} \varepsilon_{-1}
$$

for some real parameter $a$ that depends on the geometry of the six-manifold. This is the generic structure for any solution of (1.1) on a circle bundle. Here subscripts denote the mode number. Then the integral in (1.2) picks up corresponding nonzero modes from $\psi$,

$$
\delta \phi_{0}=\varepsilon_{+1} \psi_{-1}+\varepsilon_{-1} \psi_{+1}
$$

whose supersymmetry variations are

$$
\delta \psi_{ \pm 1}=\varepsilon_{ \pm 1} \partial \phi_{0} .
$$

Now let us check if the truncated Lagrangian

$$
\mathcal{L}_{5 d}=\left(\partial \phi_{0}\right)^{2}+\psi_{+1} \partial \psi_{-1}+\psi_{-1} \partial \psi_{+1}
$$

is supersymmetric. We get

$$
\delta \mathcal{L}_{5 d}=\sum \partial^{2} \phi_{0}\left(\varepsilon_{+1} \psi_{-1}+\varepsilon_{-1} \psi_{+1}\right)
$$

but this we can also write as

$$
\delta \mathcal{L}_{5 d}=\int d u \sum \partial^{2} \phi_{0} \varepsilon \psi
$$

Now let us go back to the $6 \mathrm{~d}$ Lagrangian. If we expand $\phi$ in its Fourier modes as $\phi=\sum_{n} \phi_{n} e^{i n u}$, then we get

$$
0=\delta \mathcal{L}_{6 d}=\sum \sum_{n} \partial^{2} \phi_{n} e^{i n u} \varepsilon \psi,
$$

and we know that this is zero since the $6 \mathrm{~d}$ Lagrangian is supersymmetric. Of course, if we integrate zero along the fiber, it is still zero, so we have

$$
0=\int d u \delta \mathcal{L}_{6 d}=\sum_{n} \sum \partial^{2} \phi_{n} \int d u e^{i n u} \varepsilon \psi .
$$

If we then put $\phi_{n}=0$ for all $n$ except for the zero mode $\phi_{0}$, then this reduces to

$$
0=\sum \partial^{2} \phi_{0} \int d u \varepsilon \psi=\delta \mathcal{L}_{5 d}
$$

which means that the truncated Lagrangian, where only $\phi_{0}$ is kept, is supersymmetric under the truncated supersymmetries.

This general argument fails for the non-Abelian generalization where the Lagrangian has higher order terms. For instance if the $6 \mathrm{~d}$ Lagrangian contains a cubic interaction term of the form $\phi_{+2} \psi_{-1} \psi_{-1}$ and if we have a supersymmetry variation of the form $\delta \phi_{+2}=\varepsilon_{+1} \psi_{+1}$, then the variation of that term will contain a term of the form $\varepsilon_{+1} \psi_{+1} \psi_{-1} \psi_{-1}$ that should survive if the truncation down to the modes $\phi_{0}$ and $\psi_{+1}$ were a consistent truncation. But we will never get that term if we first truncate the Lagrangian to the modes $\phi_{0}$ and $\psi_{ \pm 1}$ and then make the supersymmetry variation since then we will put the term $\phi_{+2} \psi_{-1} \psi_{-1}$ to zero in that truncated Lagrangian. So the truncation becomes inconsistent in general, when there are higher order terms. However, there can be exceptions where a truncated non-Abelian generalization can be found that is supersymmetric.

This argument also shows that the critical term to analyze in the supersymmetry variation of the non-Abelian Lagrangian will be the terms that are cubic in the fermionic fields. Typically these terms are the most difficult ones to analyze since it usually requires a Fierz rearrangement to see whether the sum of these cubic terms is zero or not. But it is really important to analyze precisely these cubic terms to see whether the non-Abelian Lagrangian is supersymmetric or not. This will become more clear as we proceed with our concrete examples.

In this paper we will study the M5 brane on $\mathbb{R} \times S^{5}$ where we have the Lorentzian time along $\mathbb{R}$. The supersymmetry parameter depends nontrivially on the time direction. First, in Sec. II, we perform dimensional reduction along the time direction and obtain a supersymmetric Abelian Lagrangian. We then show that no non-Abelian generalization exists if we insist on keeping all the supersymmetries of the Abelian theory. In Sec. II A we reduce the amount of supersymmetry and consider the smaller tensor multiplet that has just one real scalar field. Here we almost seem to find a supersymmetric non-Abelian Lagrangian in $5 \mathrm{~d}$ by using our truncation, but it turns out to fail. While most terms cancel out nicely, there are 
cubic terms in the fermionic fields that arise upon a supersymmetry variation and these have to vanish by using a Fierz rearrangement, but these terms do not vanish in that way. We then make a further Weyl projection that reduces supersymmetry further, and then finally we are able to find a supersymmetric Lagrangian. But then, in Sec. II B, we discover that if we make a simple field redefinition, our Lagrangian becomes identical with the Lagrangian that was already found in the literature on $S^{5}$ [1] and that was derived from the M5 brane in [2] by turning on an R-gauge field along the time direction.

We next consider our second example, in Sec. III, where we consider a null reduction by following closely [3]. We take our null direction as a combination of the Hopf circle on $S^{5}$ and the time direction. We first obtain the Abelian truncated theory and show that it is supersymmetric. We next show that the Abelian theory does not immediately generalize to the non-Abelian case, but if we impose further Weyl projections, then we are able to obtain a non-Abelian Lagrangian.

There are five appendixes. In particular, in Appendix A we review a $6 \mathrm{~d}$ formulation of non-Abelian 5d SYM where one introduces an auxiliary geometrical vector field [4-6] and present the closure relations that one gets for these supersymmetry variations and it was this analysis that originally led us to consider the two examples that we are presenting in this paper. Namely these two examples are following from making the two Weyl projections in Eqs. (A8) and (A9) respectively. The first Weyl projection leads us to the time reduction and the small tensor multiplet. The second Weyl projection leads us to the null reduction.

\section{M5 BRANE ON $\mathbb{R} \times S^{5}$}

The six-manifold $\mathbb{R} \times S^{5}$ can be conformally mapped to $S^{6}$ if we assume a Euclidean signature. But here we will assume a Lorentzian signature with time along the $\mathbb{R}$ direction. Our first goal is to see whether we can derive a supersymmetric theory on $S^{5}$ from an M5 brane on $\mathbb{R} \times S^{5}$ without turning on an R-gauge field along the time direction. The Abelian M5 brane on $\mathbb{R} \times S^{5}$ is well understood. In fact one can generalize to any six-manifold for which (1.1) has at least one solution. In that case we have the following supersymmetry variations:

$$
\begin{aligned}
\delta \phi^{A} & =i \bar{\varepsilon} \Gamma^{A} \psi, \\
\delta B_{M N} & =i \bar{\varepsilon} \Gamma_{M N} \psi, \\
\delta \psi & =\frac{1}{12} \Gamma^{M N P} \varepsilon H_{M N P}+\Gamma^{M} \Gamma^{A} \varepsilon \nabla_{M} \phi^{A}-4 \Gamma^{A} \eta \phi^{A},
\end{aligned}
$$

and the supersymmetric Lagrangian may be expressed as

$$
\mathcal{L}=\mathcal{L}_{B}-\frac{1}{2}\left(\nabla_{M} \phi^{A}\right)^{2}+\frac{i}{2} \bar{\psi} \Gamma^{M} \nabla_{M} \psi-\frac{R}{10}\left(\phi^{A}\right)^{2},
$$

where $\mathcal{L}_{B}$ is some Lagrangian for the self-dual tensor field whose precise form will not be very important for us now, since we will shortly reduce this Lagrangian down to five dimensions. Here $R$ is the Ricci curvature scalar on a sixmanifold. We will now specialize to $\mathbb{R} \times S^{5}$ and write the metric as

$$
d s^{2}=g_{M N} d x^{M} d x^{N}=-d t^{2}+G_{m n} d x^{m} d x^{n} .
$$

To reduce down to $S^{5}$, we will represent the gamma matrices in terms of five-dimensional gamma matrices $\gamma^{m}$ and $\tau^{A}$ as follows:

$$
\begin{aligned}
\Gamma^{t} & =i \sigma^{2} \otimes 1 \otimes 1, \\
\Gamma^{m} & =\sigma^{1} \otimes \gamma^{m} \otimes 1, \\
\Gamma^{A} & =\sigma^{3} \otimes 1 \otimes \tau^{A} .
\end{aligned}
$$

The $6 \mathrm{~d}$ chirality matrix is

$$
\Gamma=\sigma^{3} \otimes 1 \otimes 1
$$

and $\varepsilon$ and $\psi$ have opposite chiralities

$$
\Gamma \varepsilon=-\varepsilon, \quad \Gamma \psi=\psi,
$$

and they are Majorana spinors in 11 dimensions,

$$
\bar{\varepsilon}=\varepsilon^{T} C_{11 d}, \quad \bar{\psi}=\psi^{T} C_{11 d},
$$

where the Dirac conjugate is defined as $\bar{\psi}=\psi^{\dagger} \Gamma^{t}$. We may solve (1.1) by separating its components as

$$
\partial_{t} \varepsilon=\Gamma_{t} \eta, \quad \nabla_{m} \varepsilon=\Gamma_{m} \eta .
$$

We use the relation

$$
\Gamma^{m n} \nabla_{m} \nabla_{n} \varepsilon=-\frac{R}{4} \varepsilon
$$

where $R=\frac{20}{r^{2}}$ is the Ricci scalar on $S^{5}$ with radius $r$, to find the solution

$$
\mathcal{E}=e^{\frac{i}{2 r} t}\left(\begin{array}{l}
0 \\
\mathcal{E}
\end{array}\right)+e^{-\frac{i}{2 r} t}\left(\begin{array}{l}
0 \\
\mathcal{F}
\end{array}\right) .
$$

We also get

$$
\eta=\frac{i}{2 r} e^{\frac{i}{2 r} t}\left(\begin{array}{l}
\mathcal{E} \\
0
\end{array}\right)-\frac{i}{2 r} e^{-\frac{i}{2 r} t}\left(\begin{array}{l}
\mathcal{F} \\
0
\end{array}\right) .
$$

Here

$$
\nabla_{m} \mathcal{E}=\frac{i}{2 r} \gamma_{m} \mathcal{E}, \quad \nabla_{m} \mathcal{F}=-\frac{i}{2 r} \gamma_{m} \mathcal{F} .
$$


Perhaps the best way to see that this solves (1.1) is by simply plugging in this solution into (2.1) to see that these equations are both satisfied. Let us now study the Majorana condition more closely. The 11-dimensional charge conjugation matrix is antisymmetric,

$$
C_{11 d}^{T}=-C_{11 d},
$$

and we will represent it as

$$
C_{11 d}=\varepsilon \otimes C \otimes \tilde{C},
$$

where $C$ and $\tilde{C}$ are antisymmetric charge conjugation matrices in $5 \mathrm{~d}$, and $\varepsilon$ is the antisymmetric tensor. At this point, things get clearer when we write out all the spinor indices explicitly though, so let us do that here,

$$
\left(C_{11 d}\right)_{a \alpha \dot{\alpha}, b \beta \dot{\beta}}=\varepsilon_{a b} C_{\alpha \beta} C_{\dot{\alpha} \dot{\beta}} .
$$

Then the Majorana condition becomes

$$
\left(\psi^{a \alpha \dot{\alpha}}\right)^{*} i\left(\sigma^{2}\right)_{b}^{a}=\psi^{a \dot{\beta} \dot{\beta}} \varepsilon_{a b} C_{\beta \alpha} C_{\dot{\beta} \dot{\alpha}} .
$$

We will define the antisymmetric tensor $\varepsilon_{a b}$ such that

$$
\varepsilon_{+-}=1
$$

and then we get

$$
\left(\psi^{+\alpha \dot{\alpha}}\right)^{*}=\psi^{+\beta \dot{\beta}} C_{\beta \alpha} C_{\dot{\beta} \dot{\alpha}}, \quad\left(\varepsilon^{-\alpha \dot{\alpha}}\right)^{*}=\varepsilon^{-\beta \dot{\beta}} C_{\beta \alpha} C_{\dot{\beta} \dot{\alpha}} .
$$

From now on we will drop the $6 \mathrm{~d}$ chirality indices \pm as they play no significant role in $5 \mathrm{~d}$. In $5 \mathrm{~d}$ we do not really have a Majorana condition for the nonzero modes. What we have instead is a relation between $\mathcal{E}$ and $\mathcal{F}$,

$$
\left(\mathcal{E}^{\alpha \dot{\alpha}}\right)^{*}=C_{\alpha \beta} C_{\dot{\alpha} \dot{\beta}} \mathcal{F}^{\beta \dot{\beta}}, \quad\left(\mathcal{F}^{\alpha \dot{\alpha}}\right)^{*}=C_{\alpha \beta} C_{\dot{\alpha} \dot{\beta}} \mathcal{E}^{\beta \dot{\beta}} .
$$

These relations follow easily from using the explicit form of our solution, Eq. (2.2). But now we would also like to derive the second condition from the first one by taking the complex conjugate. Taking the complex conjugate of the first equation, we get

$$
\mathcal{E}^{\alpha \dot{\alpha}}=\left(C_{\alpha \beta}\right)^{*}\left(C_{\dot{\alpha} \dot{\beta}}\right)^{*}\left(\mathcal{F}^{\beta \dot{\beta}}\right)^{*} .
$$

We may now multiply by charge conjugation matrices on both sides to get

$$
C_{\alpha \beta} C_{\dot{\alpha} \dot{\beta}} \mathcal{E}^{\dot{\beta} \dot{\beta}}=C_{\alpha \beta} C_{\dot{\alpha} \dot{\beta}}\left(C_{\beta \gamma}\right)^{*}\left(C_{\dot{\beta} \dot{\gamma}}\right)^{*}\left(\mathcal{F}^{\gamma \dot{\gamma}}\right)^{*} .
$$

We shall require that

$$
C_{\alpha \beta}\left(C_{\beta \gamma}\right)^{*}=-\delta_{\alpha}^{\gamma}
$$

The reason why we put the minus sign here will become clear later on. We can now introduce the inverse

$$
C^{\gamma \beta}=\left(C_{\beta \gamma}\right)^{*} .
$$

We use $C_{\alpha \beta}$ and $C^{\alpha \beta}$ to lower and rise spinor indices by always acting from the left,

$$
\psi_{\alpha}=C_{\alpha \beta} \psi^{\beta}, \quad \psi^{\alpha}=C^{\alpha \beta} \psi_{\beta} .
$$

So we define for example

$$
\left(\gamma^{m}\right)^{\alpha \beta}=C^{\beta \gamma}\left(\gamma^{m}\right)^{\alpha}{ }_{\gamma} .
$$

We may now find the following relations:

$$
C^{\alpha}{ }_{\beta}=C^{\alpha \gamma} C_{\gamma \beta}=\delta_{\beta}^{\alpha}, \quad C_{\alpha}^{\beta}=C^{\beta \gamma} C_{\alpha \gamma}=-\delta_{\beta}^{\alpha} .
$$

We have the Fierz expansion of two anticommuting spinors,

$$
\psi^{\alpha} \psi^{\beta}=A C^{\alpha \beta}+B_{m}\left(\gamma^{m}\right)^{\alpha \beta}+C_{m n}\left(\gamma^{m n}\right)^{\alpha \beta} .
$$

It corresponds to the following expansion of the tensor product of two spinor representations:

$$
4 \otimes 4=1_{a} \oplus 5_{a} \oplus 10_{s} .
$$

The subscripts $a$ and $s$ stand for antisymmetric and symmetric representations, so we must have that $C^{\alpha \beta}$ and $\left(\gamma^{m}\right)^{\alpha \beta}$ are antisymmetric, whereas $\left(\gamma^{m n}\right)^{\alpha \beta}$ is symmetric in $\alpha$ and $\beta$. Our 5d spinor notations follow closely Ref. [1].

The time direction in Euclidean $\mathbb{R} \times S^{5}$ is noncompact if this shall be related by a conformal map to $S^{6}$. But in Lorentzian signature that we will consider here, the time direction can be taken to be a compact circle with radius $2 \pi r$. We will refrain from discussing any physical implications of having a compact time direction. From a purely mathematical viewpoint of classical supersymmetric field theory, having a compact time direction simply means that we may expand the fields in Fourier modes in the time direction by assuming that time has a periodicity $t \sim t+2 \pi r$. For fermions there is as always a possibility of having either periodic or antiperiodic boundary conditions. Since the supersymmetry parameter depends on time through the exponential factors $e^{ \pm \frac{i}{2 r} t}$ which is antiperiodic as $t$ goes to $t+2 \pi r$, we conclude that fermions shall have antiperiodic boundary conditions if we want to have a supersymmetric theory. The bosonic fields must be periodic and therefore only even modes are kept for the bosonic fields, whereas for the fermionic field only the odd modes are kept. And if only the odd modes are kept, it means that there is no fermionic zero mode present. 
But we do not think that we will be able to find a nonAbelian theory if we keep infinitely many Kaluza-Klein modes, neither do we think this is really the right thing to do when the gauge group is non-Abelian because then we shall have instanton particles that are expected to fill in missing modes when we truncate the modes to a finite number of modes. Now instead of truncating to the fermionic zero modes as one normally does in usual dimensional reduction, we will truncate to the lowest lying odd Fourier modes

$$
\psi=e^{\frac{i}{2 r} t}\left(\begin{array}{l}
\chi \\
0
\end{array}\right)+e^{-\frac{i}{2 r} t}\left(\begin{array}{l}
\zeta \\
0
\end{array}\right) .
$$

Then the fermionic field has the same type of expansion as the supersymmetry parameter $\varepsilon$ and there is a chance that this will preserve some supersymmetry. There is no Majorana condition on these modes but instead there is a relation between the two modes,

$$
\left(\chi^{\alpha \dot{\alpha}}\right)^{*}=C_{\alpha \beta} C_{\dot{\alpha} \dot{\beta}} \beta^{\beta \dot{\beta}} .
$$

The supersymmetry variations can be derived easily by truncating the supesymmetry variations for the Abelian M5 brane. We get

$$
\begin{aligned}
\delta \phi^{A} & =-i \mathcal{E}^{\dagger} \tau^{A} \chi-i \mathcal{F}^{\dagger} \tau^{A} \zeta, \\
\delta A_{m} & =-i \mathcal{E}^{\dagger} \gamma_{m} \chi-i \mathcal{F}^{\dagger} \gamma_{m} \zeta, \\
\delta \chi & =\frac{1}{2} \gamma^{m n} \mathcal{E} F_{m n}-\gamma^{m} \tau^{A} \mathcal{E} \nabla_{m} \phi^{A}-\frac{2 i}{r} \tau^{A} \mathcal{E} \phi^{A}, \\
\delta \zeta & =\frac{1}{2} \gamma^{m n} \mathcal{F} F_{m n}-\gamma^{m} \tau^{A} \mathcal{F} \nabla_{m} \phi^{A}+\frac{2 i}{r} \tau^{A} \mathcal{F} \phi^{A} .
\end{aligned}
$$

The corresponding supersymmetric Lagrangian is given by

$$
\begin{aligned}
\mathcal{L}= & \frac{1}{4} F_{m n}^{2}-\frac{1}{2}\left(\nabla_{m} \phi^{A}\right)^{2}+\frac{i}{2} \chi^{\dagger} \gamma^{m} \nabla_{m} \chi+\frac{i}{2} \zeta^{\dagger} \gamma^{m} \nabla_{m} \zeta \\
& -\frac{2}{r^{2}}\left(\phi^{A}\right)^{2}+\frac{1}{4 r}\left(\chi^{\dagger} \chi-\zeta^{\dagger} \zeta\right) .
\end{aligned}
$$

The natural choice is to take $\varepsilon$ to be an anticommuting parameter. In that case the variations of the bosonic fields become Hermitian, and we may write these variations as

$$
\begin{aligned}
\delta \phi^{A} & =-i \mathcal{E}^{\dagger} \tau^{A} \chi+i \chi^{\dagger} \tau^{A} \mathcal{E}, \\
\delta A_{m} & =-i \mathcal{E}^{\dagger} \gamma_{m} \chi+i \chi^{\dagger} \gamma_{m} \mathcal{E}, \\
\delta \chi & =\frac{1}{2} \gamma^{m n} \mathcal{E} F_{m n}-\gamma^{m} \tau^{A} \mathcal{E} \partial_{m} \phi^{A}-\frac{2 i}{r} \tau^{A} \mathcal{E} \phi^{A} .
\end{aligned}
$$

We may also write the Lagrangian as

$$
\begin{aligned}
\mathcal{L}= & \frac{1}{4} F_{m n}^{2}-\frac{1}{2}\left(\nabla_{m} \phi^{A}\right)^{2}+i \chi^{\dagger} \gamma^{m} \nabla_{m} \chi \\
& -\frac{2}{r^{2}}\left(\phi^{A}\right)^{2}+\frac{1}{2 r} \chi^{\dagger} \chi .
\end{aligned}
$$

One may now easily verify that this Lagrangian is invariant under these supersymmetry variations by just using the Killing spinor equation

$$
\nabla_{m} \mathcal{E}=\frac{i}{2 r} \gamma_{m} \mathcal{E}
$$

This result is encouraging because it provides our first example of a dimensionally reduced theory that has supersymmetry although the 6d theory has a supersymmetry parameter that depends nontrivially on the circle along which we reduce. Having a supersymmetric Lagrangian, we may also expect that these supersymmetry variations close on some symmetry variations of the Lagrangian.

However, we will now see that no non-Abelian generalization of this Abelian Lagrangian can be constructed that is supersymmetric. To show this we will proceed iteratively. First we just replace all the derivatives $\nabla_{m}$ with gauge covariant derivatives $D_{m}=\nabla_{m}-i\left[A_{m}, \bullet\right]$ and assume all fields are in the adjoint representation. Then of course the Lagrangian will not be supersymmetric. We then find correction terms such that we cancel the unwanted terms, but such correction terms will also generate new terms that we also need to cancel by adding further correction terms. This can be analysed fairly systematically. In the end, we will find a fully corrected Lagrangian and corresponding supersymmetry variations but still that Lagrangian will not be supersymmetric. Because of the apparent uniqueness of each term we find in each iteration step, we consider this to be a no-go proof.

First, if we just replace $\nabla_{m}$ with $D_{m}$ everywhere, then we get the following nonvanishing variation of the Lagrangian:

$$
\delta \mathcal{L}=-\frac{1}{2} \chi^{\dagger} \gamma^{m n} \tau^{A} \mathcal{E}\left[F_{m n}, \phi^{A}\right]-\chi^{\dagger} \gamma^{m} \mathcal{E}\left[\phi^{A}, D_{m} \phi^{A}\right]
$$

where we define the gauge covariant derivative so that

$$
\left[D_{m}, D_{n}\right] \phi=-i\left[F_{m n}, \phi\right] .
$$

We next cancel both these terms by adding to the Lagrangian the following coupling term:

$$
\mathcal{L}_{1}=\chi^{\dagger} \tau^{A}\left[\chi, \phi^{A}\right]
$$

We cannot imagine any other term that can do this job. But by adding this term, some new terms will be generated as well, and so now we get 
$\delta \mathcal{L}+\delta \mathcal{L}_{1}=\frac{1}{2} \chi^{\dagger} \tau^{A B} \gamma^{m} \mathcal{E} D_{m}\left(\left[\phi^{A}, \phi^{B}\right]\right)+\frac{2 i}{r} \chi^{\dagger} \tau^{A B} \mathcal{E}\left[\phi^{A}, \phi^{B}\right]$

plus some cubic terms in $\chi$ that we will not need to analyze further here. Now these two terms can be canceled by modifying the supersymmetry variation by adding the term

$$
\delta_{1} \chi=\frac{i}{2} \tau^{A B} \mathcal{E}\left[\phi^{A}, \phi^{B}\right]
$$

to $\delta \chi$. But that will also generate another term

$$
\delta_{1} \mathcal{L}_{1}=i \chi^{\dagger} \tau^{C} \mathcal{E}\left[\left[\phi^{A}, \phi^{C}\right], \phi^{C}\right]
$$

but that we can easily cancel by adding the term

$$
\mathcal{L}_{2}=-\frac{1}{4}\left[\phi^{A}, \phi^{B}\right]^{2}
$$

But even when taking into account all these non-Abelian correction terms, we will still end up with a nonvanishing variation

$$
\begin{aligned}
\left(\delta+\delta_{1}\right)\left(\mathcal{L}+\mathcal{L}_{1}\right) & =\left(-\frac{5 i}{4 r}+\frac{i}{4 r}+\frac{2 i}{r}\right) \chi^{\dagger} \tau^{A B} \mathcal{E}\left[\phi^{A}, \phi^{B}\right] \\
& =\frac{i}{r} \chi^{\dagger} \tau^{A B} \mathcal{E}\left[\phi^{A}, \phi^{B}\right]
\end{aligned}
$$

plus those cubic terms in the fermionic fields that we did not analyze here since it is already clear that no nonAbelian Lagrangian can be found. There now is no further terms that we can add that could cancel this nonvanishing variation. This finishes our no-go proof.

\section{A. The small vector multiplet}

We may be more successful with finding a non-Abelian generalization if we make our tensor multplet smaller. To this end we will impose the Weyl projection

$$
\tau^{5} \mathcal{E}=\mathcal{E}
$$

on the supersymmetry parameter, thus reducing the amount of supersymmetry by half. This will reduce the R-symmetry as $S O(5) \rightarrow S U(2)_{R}$. But of course, by selecting the fifth direction in (2.2), we will just break $S O(5) \rightarrow S O(4)=S U(2)_{F} \times S U(2)_{R}$ but the $S U(2)_{F}$ will not rotate the supercharges, it will be a flavor symmetry. The original Abelian tensor multiplet breaks into one smaller tensor multiplet with just one real scalar field $\phi=\phi^{5}$ and a fermionic field that is also subject to the Weyl projection

$$
\tau^{5} \psi=\psi .
$$

Then the remaining fields are four real scalars, and another fermionic field subject to the opposite Weyl projection $\tau^{5} \psi=-\psi$. These fields form a hypermultiplet. We will discard this hypermultiplet and only focus on the small tensor multiplet.

Let us now introduce some index notations for the $\mathrm{R}$-symmery. We denote a spinor as

$$
\psi^{\alpha \dot{\alpha}}=\left(\begin{array}{c}
\psi_{I}^{\alpha} \\
\psi^{\alpha A}
\end{array}\right) .
$$

The flavor index $A$ is a two-component spinor index that shall not be confused with the $S O(5)$ vector index $A$. We define the gamma matrices $\tau^{A}=\left(\tau^{i}, \tau^{5}\right)$ as

$$
\tau^{i}=\left(\begin{array}{cc}
0 & \sigma_{I B}^{i} \\
\sigma^{i, A J} & 0
\end{array}\right), \quad \tau^{5}=\left(\begin{array}{cc}
\delta_{I}^{J} & 0 \\
0 & -\delta_{B}^{A}
\end{array}\right) .
$$

The supersymmetry parameter that satisfies $\tau^{5} \mathcal{E}=\mathcal{E}$ has a nonvanishing component $\mathcal{E}_{I}$,

$$
\mathcal{E}=\left(\begin{array}{c}
\mathcal{E}_{I} \\
0
\end{array}\right) .
$$

The antisymmetric charge conjugation matrix is represented as

$$
C_{\dot{\alpha} \dot{\beta}}=\left(\begin{array}{cc}
\varepsilon^{I J} & 0 \\
0 & \varepsilon_{A B}
\end{array}\right)
$$

We have

$$
\left(\mathcal{E}_{I}^{\alpha}\right)^{*}=C_{\alpha \beta} \varepsilon^{I J} \mathcal{F}_{J}^{\beta}, \quad\left(\chi_{I}^{\alpha}\right)^{*}=C_{\alpha \beta} \varepsilon^{I J} \zeta_{J}^{\beta} .
$$

The Killing spinor equations are

$$
\nabla_{m} \mathcal{E}_{I}=\frac{i}{2 r} \gamma_{m} \mathcal{E}_{I}, \quad \nabla_{m} \mathcal{F}_{I}=-\frac{i}{2 r} \gamma_{m} \mathcal{F}_{I},
$$

where the second equation can be obtained from the first by complex conjugation.

The supersymmetry variations for the small tensor multiplet are

$$
\begin{aligned}
\delta \phi & =-i\left(\mathcal{E}_{I}\right)^{\dagger} \chi_{I}-i\left(\mathcal{F}_{I}\right)^{\dagger} \zeta_{I}, \\
\delta A_{m} & =-i\left(\mathcal{E}_{I}\right)^{\dagger} \gamma_{m} \chi_{I}-i\left(\mathcal{F}_{I}\right)^{\dagger} \gamma_{m} \zeta_{I}, \\
\delta \chi_{I} & =\frac{1}{2} \gamma^{m n} \mathcal{E}_{I} F_{m n}-\gamma^{m} \mathcal{E}_{I} D_{m} \phi-\frac{2 i}{r} \mathcal{E}_{I} \phi .
\end{aligned}
$$

and the supersymmetric Lagrangian is

$$
\begin{aligned}
\mathcal{L}= & \frac{1}{4} F_{m n}^{2}-\frac{1}{2}\left(D_{m} \phi\right)^{2}-\frac{2}{r^{2}} \phi^{2} \\
& +i\left(\chi_{I}\right)^{\dagger} \gamma^{m} D_{m} \chi_{I}+\frac{1}{2 r}\left(\chi_{I}\right)^{\dagger} \chi_{I} .
\end{aligned}
$$

The closure relations for these supersymmetry variations are nonstandard, 


$$
\begin{aligned}
{\left[\delta_{2}, \delta_{1}\right] \phi=} & 2 i \mathcal{L}_{v} \phi, \\
{\left[\delta_{2}, \delta_{1}\right] A_{m}=} & 2 i \mathcal{L}_{v} A_{m}+D_{m} \Lambda, \\
{\left[\delta_{2}, \delta_{1}\right] \chi_{I}=} & 8 i \mathcal{L}_{B} \chi_{I}+\frac{12}{r} A_{I}^{J} \chi_{J}-i\left(3{A_{I}^{J}}^{J}+3 B_{p I}^{J} \gamma^{p}-C_{p q I}^{J} \gamma^{p q}\right)\left(\gamma^{m} \nabla_{m} \chi_{J}+\frac{1}{2 r} \chi_{J}\right) \\
& +8 i \mathcal{L}_{\tilde{B}} \chi_{I}-\frac{16}{r} \tilde{A}_{I}^{J} \zeta_{J}-\frac{4}{r} \tilde{B}_{m I}^{J} \gamma^{m} \zeta_{J}-i\left(3 \tilde{A}_{I}^{J}+3 \tilde{B}_{p I}^{J} \gamma^{p}-\tilde{C}_{p q I}^{J} \gamma^{p q}\right)\left(\gamma^{m} \nabla_{m} \zeta_{J}-\frac{1}{2 r} \zeta_{J}\right),
\end{aligned}
$$

where

$$
\begin{aligned}
\mathcal{L}_{B} \chi_{I} & :=B^{m}{ }_{I}^{J} \nabla_{m} \chi_{J}+\frac{1}{4} \nabla_{m} B_{n I}{ }^{J} \gamma^{m n} \chi_{J} \\
& =B^{m}{ }_{I}^{J} \nabla_{m} \chi_{J}+\frac{i}{2 r} C_{m n I}{ }^{J} \gamma^{m n} \chi_{J}, \\
\mathcal{L}_{\tilde{B}} \zeta_{I} & :=\tilde{B}_{I}^{m}{ }_{I}^{J} \nabla_{m} \zeta_{J}+\frac{1}{4} \nabla_{m} \tilde{B}_{n I}{ }^{J} \gamma^{m n} \zeta_{J}=\tilde{B}_{I}^{m}{ }_{I}^{J} \nabla_{m} \zeta_{J} .
\end{aligned}
$$

Here the various coefficients are defined as

$$
\begin{aligned}
\mathcal{E}_{I}\left(\mathcal{E}_{J}\right)^{\dagger}=A^{J}{ }_{I}+B_{m}{ }^{J}{ }_{I} \gamma^{m}+C_{m n}{ }^{J}{ }_{I} \gamma^{m n}, \\
\mathcal{E}_{I}\left(\mathcal{F}_{J}\right)^{\dagger}=\tilde{A}^{J}{ }_{I}+\tilde{B}_{m}{ }^{J}{ }_{I} \gamma^{m}+\tilde{C}_{m n}{ }^{J}{ }_{I} \gamma^{m n},
\end{aligned}
$$

where

$$
\begin{aligned}
A^{J}{ }_{I} & =-\frac{1}{4}\left(\mathcal{E}_{J}\right)^{\dagger} \mathcal{E}_{I}, \\
B_{m}{ }^{J} & =-\frac{1}{4}\left(\mathcal{E}_{J}\right)^{\dagger} \gamma_{m} \mathcal{E}_{I}, \\
C_{m n}{ }^{J} & =\frac{1}{8}\left(\mathcal{E}_{J}\right)^{\dagger} \gamma_{m n} \mathcal{E}_{I},
\end{aligned}
$$

and

$$
\begin{aligned}
\tilde{A}^{J}{ }_{I} & =-\frac{1}{4}\left(\mathcal{F}_{J}\right)^{\dagger} \mathcal{E}_{I}, \\
\tilde{B}_{m}{ }^{J} & =-\frac{1}{4}\left(\mathcal{F}_{J}\right)^{\dagger} \gamma_{m} \mathcal{E}_{I}, \\
\tilde{C}_{m n}{ }^{J}{ }^{J} & =\frac{1}{8}\left(\mathcal{F}_{J}\right)^{\dagger} \gamma_{m n} \mathcal{E}_{I} .
\end{aligned}
$$

There are the following differential relations between these coefficients that one may derive by using the Killing spinor equations,

$$
\begin{aligned}
\nabla_{m} A^{J}{ }_{I} & =0, \\
\nabla_{m} B_{m}{ }^{J}{ } & =\frac{2 i}{r} C_{m n}{ }^{J}, \\
\nabla_{m} \tilde{A}^{J}{ }_{I} & =-\frac{i}{r} \tilde{B}_{m}{ }_{I}{ }_{I}, \\
\nabla_{m} \tilde{B}_{n}{ }^{J}{ }_{I} & =\frac{i}{r} \tilde{A}^{J}{ }_{I} G_{m n} .
\end{aligned}
$$

These closure relations reflect the fact that there are many more fermionic degrees of freedom than there are bosonic ones, so closure on the fermion does not give back the same fermion translated or gauge transformed, but instead it maps us back into a linear combination of $\chi_{I}$ and $\zeta_{I}$.

Let us now turn our attention to the non-Abelian generalization. The only candidate Lagrangian appears to be

$$
\begin{aligned}
\mathcal{L}= & \frac{1}{4} F_{m n}^{2}-\frac{1}{2}\left(D_{m} \phi\right)^{2}-\frac{2}{r^{2}} \phi^{2} \\
& +i\left(\chi_{I}\right)^{\dagger} \gamma^{m} D_{m} \chi_{I}+\frac{1}{2 r}\left(\chi_{I}\right)^{\dagger} \chi_{I}+e\left(\chi_{I}\right)^{\dagger}\left[\chi_{I}, \phi\right]
\end{aligned}
$$

since its supersymmetry variation is given by $\delta \mathcal{L}=T$ where $T$ is used to denote the cubic terms in the fermionic fields,

$$
\begin{aligned}
T & :=e\left(\chi_{I}\right)^{\dagger}\left[\chi_{I}, \delta \phi\right]+i\left(\chi_{I}\right)^{\dagger} \gamma^{m}(-i e)\left[\delta A_{m}, \chi_{I}\right] \\
& =-i e\left(\mathcal{E}_{J}^{\gamma}\right)^{*}\left[\chi_{J}^{\gamma c}\left(\chi_{I}^{\beta a}\right)^{*}-\left(\gamma_{m}\right)^{\gamma} \chi_{J}^{\delta c}\left(\chi_{I}^{\alpha a}\right)^{*}\left(\gamma^{m}\right)^{\alpha}{ }_{\beta}\right] \chi_{I}^{\beta b} .
\end{aligned}
$$

We should then ask whether $T$ is vanishing by a Fierz rearrangement. It turns out to not vanish, so the Lagrangian is not supersymmetric and therefore we conclude that no non-Abelian generalization exists with this amount of supersymmetry.

We can reduce the amount of supersymmetry so that the R-symmetry is further reduced from $S U(2)_{R}$ down to $U(1)_{R}$ by imposing the Weyl condition

$$
\left(\sigma^{3}\right)_{I}^{J} \mathcal{E}_{J}=\mathcal{E}_{J}
$$

Then there is just one complex supersymmetry parameter $\mathcal{E}=\mathcal{E}_{1}$. With this projection, one finds that the component $\chi_{2}$ does not enter the supersymmetry multiplet as its supersymmetry variation becomes zero,

$$
\delta \chi_{2}=0,
$$

and so we define $\chi:=\chi_{1}$ for which we find the supersymmetry variations 


$$
\begin{aligned}
\delta \phi & =-i \mathcal{E}^{\dagger} \chi-i \mathcal{F}^{\dagger} \zeta, \\
\delta A_{m} & =-i \mathcal{E}^{\dagger} \gamma_{m} \chi-i \mathcal{F}^{\dagger} \gamma_{m} \zeta, \\
\delta \chi & =\frac{1}{2} \gamma^{m n} \mathcal{E} F_{m n}-\gamma^{m} \mathcal{E} D_{m} \phi-\frac{2 i}{r} \mathcal{E} \phi .
\end{aligned}
$$

The Lagrangian is ${ }^{1}$

$$
\begin{aligned}
\mathcal{L}= & \frac{1}{4} F_{m n}^{2}-\frac{1}{2}\left(D_{m} \phi\right)^{2}-\frac{2}{r^{2}} \phi^{2} \\
& +i \chi^{\dagger} \gamma^{m} D_{m} \chi+\frac{1}{2 r} \chi^{\dagger} \chi+e \chi^{\dagger}[\chi, \phi] .
\end{aligned}
$$

The Killing spinor equation is

$$
\nabla_{m} \mathcal{E}=\frac{i}{2 r} \gamma_{m} \mathcal{E}
$$

Originally we had

$$
\mathcal{F}_{2}^{\alpha}=\varepsilon_{21} C^{\alpha \beta}\left(\mathcal{E}_{1}^{\beta}\right)^{*}, \quad \zeta_{2}^{\alpha}=\varepsilon_{21} C^{\alpha \beta}\left(\chi_{1}^{\beta}\right)^{*} .
$$

Now we define $\mathcal{F}^{\alpha}:=\mathcal{F}_{2}^{\alpha}$ and $\zeta^{\alpha}:=\zeta_{2}^{\alpha}$ so with $\varepsilon^{12}=1$, we get the relations

$$
\mathcal{F}^{\alpha}=C^{\alpha \beta}\left(\mathcal{E}^{\beta}\right)^{*}, \quad \zeta^{\alpha}=C^{\alpha \beta}\left(\chi^{\beta}\right)^{*} .
$$

Let us now again analyze the cubic terms in the fermionic field that arise upon a supersymmetry variation of this Lagrangian. These terms are

$$
\begin{aligned}
T & :=e(\chi)^{\dagger}[\chi, \delta \phi]+i(\chi)^{\dagger} \gamma^{m}(-i e)\left[\delta A_{m}, \chi\right], \\
& =-i e\left(\mathcal{E}^{\gamma}\right)^{*}\left[\chi^{\gamma c}\left(\chi^{\beta a}\right)^{*}-\left(\gamma_{m}\right)^{\gamma} \chi^{\delta c}\left(\chi^{\alpha a}\right)^{*}\left(\gamma^{m}\right)_{\beta}^{\alpha}\right] \chi^{\beta b} .
\end{aligned}
$$

We expand

$$
\chi^{\alpha a}\left(\chi^{\beta b}\right)^{*}=\delta_{\beta}^{\alpha} A^{a b}+\left(\gamma^{m}\right)_{\beta}^{\alpha} B_{m}^{a b}+\left(\gamma^{m n}\right)_{\beta}^{\alpha} C_{m n}^{a b}
$$

and then

$$
T=4 i e\left(\mathcal{E}^{\gamma}\right)^{*}\left[\delta_{\beta}^{\gamma} A^{c a}-\left(\gamma^{m}\right)_{\beta}^{\gamma} B_{m}^{c a}\right] \chi^{\beta b}
$$

Here

${ }^{1}$ However, we still have the Lagrangian for $\chi_{2}$ as well,

$$
\mathcal{L}_{2}=i\left(\chi_{2}\right)^{\dagger} \gamma^{m} D_{m} \chi_{2}+\frac{1}{2 r}\left(\chi_{2}\right)^{\dagger} \chi_{2}+e\left(\chi_{2}\right)^{\dagger}\left[\chi_{2}, \phi\right],
$$

but this Lagrangian is not supersymmetric since the corresponding cubic term $T$ upon a supersymmetry variation will not be vanishing, but it is now consistent with supersymmetry to truncate to $\chi_{2}=0$ since the supersymmetry variation of $\chi_{2}$ is vanishing. So then we will simply get $\mathcal{L}_{2}=0$ and we retain supersymmetry of $\mathcal{L}_{2}$ trivially by putting $\chi_{2}=0$ as a truncation that is consistent with supersymmetry.

$$
\begin{aligned}
& A^{c a}=-\frac{1}{4}\left(\chi^{\alpha a}\right)^{*} \chi^{\alpha c}, \\
& B_{m}^{c a}=-\frac{1}{4}\left(\chi^{\alpha a}\right)^{*}\left(\gamma_{m}\right)^{\alpha}{ }_{\beta} \chi^{\beta c} .
\end{aligned}
$$

So we have

$$
\begin{aligned}
T= & i e\left(\mathcal{E}^{\gamma}\right)^{*} \chi^{\gamma c}\left(\chi^{\alpha a}\right)^{*} \chi^{\alpha b} \\
& -i e\left(\mathcal{E}^{\gamma}\right)^{*}\left(\gamma^{m}\right)^{\gamma}{ }_{\beta} \chi^{\beta c}\left(\chi^{\delta a}\right)^{*}\left(\gamma_{m}\right)^{\delta}{ }_{\epsilon} \chi^{\epsilon b} .
\end{aligned}
$$

We now see that we got back the same expression as the one we started with, but with an overall minus sign, so $T=-T$, which clearly shows that $T=0$ and the Lagrangian is supersymmetric.

\section{B. A dual description with an R-gauge field}

By making a few changes of viewpoint we may recover the theory one gets by turing on an R-gauge field and make contact with the results in [1]. We relabel the spinor field and its complex conjugate field as

$$
\chi=\psi_{1}, \quad \zeta=\psi_{2},
$$

and similarly

$$
\mathcal{E}=\mathcal{E}_{1}, \quad \mathcal{F}=\mathcal{E}_{2} .
$$

Then we may state a Majorana condition as

$$
\psi_{I}^{\alpha}=\varepsilon_{I J} C^{\alpha \beta}\left(\psi_{J}^{\beta}\right)^{*}
$$

that we get from

$$
\zeta^{\alpha}=C^{\alpha \beta}\left(\chi^{\beta}\right)^{*} .
$$

Moreover, the Killing spinor equations for $\chi$ and $\zeta$ can now be grouped together into one Killing spinor equation for the Majorana spinor $\mathcal{E}_{I}$

$$
\nabla_{m} \mathcal{E}_{I}=\frac{i}{2 r}\left(\sigma^{3}\right)_{I}^{J} \gamma_{m} \mathcal{E}_{J}
$$

So there is a map between the theory we get by turning on an R-gauge field, and the theory we get in this entirely different way by keeping nonzero modes for the fermionic field and not turning on any R-gauge field.

In one viewpoint, $\chi$ and $\zeta$ are nonzero Kaluza-Klein modes who receive an extra mass simply by the fact that they are nonzero modes. In the other viewpoint, $\chi$ and $\zeta$ form two components in an $S U(2)_{R}$ Majorana spinor which is a zero mode spinor upon dimensional reduction with an $\mathrm{R}$-gauge field turned on and the mass of these fermions is induced from that R-gauge field in the six-dimensional theory. Both ways result in the same $5 \mathrm{~d}$ theory, but the $6 \mathrm{~d}$ theories seem to be very different. 
Once having realized this kind of dual description, we can proceed and use all knowledge that we already have of this $5 \mathrm{~d}$ theory from say [1]. Following [1], we may take the supersymmetry variations off-shell,

$$
\begin{aligned}
\delta \phi= & -i\left(\mathcal{E}_{I}\right)^{\dagger} \psi_{I}, \\
\delta A_{m}= & -i\left(\mathcal{E}_{I}\right)^{\dagger} \gamma_{m} \psi_{I}, \\
\delta \psi_{I}= & \frac{1}{2} \gamma^{m n} \mathcal{E}_{I} F_{m n}-\gamma^{m} \mathcal{E}_{I} \partial_{m} \phi-\frac{i}{r}\left(\sigma^{3}\right)_{I}{ }^{J} \mathcal{E}_{J} \phi+\mathcal{E}_{J} D^{J}{ }_{I}, \\
\delta D_{I}^{J}= & 2\left(\mathcal{E}_{J}\right)^{\dagger}\left(i \gamma^{m} \nabla_{m} \psi_{I}+\frac{1}{2 r}\left(\sigma^{3}\right)_{I}{ }_{I} \psi_{L}\right) \\
& -\frac{1}{r}\left(\sigma^{3}\right)_{I}^{J}\left(\mathcal{E}_{K}\right)^{\dagger} \psi_{K} \\
& -\delta_{I}^{J}\left(\mathcal{E}_{K}\right)^{\dagger}\left(i \gamma^{m} \nabla_{m} \psi_{K}+\frac{1}{2 r}\left(\sigma^{3}\right)_{K}{ }^{L} \psi_{L}\right),
\end{aligned}
$$

where the second line in the variation of $D^{J}{ }_{I}$ removes the trace part, where we notice that $\sigma^{3}$ is already traceless. The Lagrangian is

$$
\begin{aligned}
\mathcal{L}= & \frac{1}{4} F_{m n}^{2}-\frac{1}{2}\left(\nabla_{m} \phi\right)^{2} \\
& +\frac{1}{4} D^{I}{ }_{J} D_{I}^{J}+\frac{i}{2 r}\left(\sigma^{3}\right)_{I}^{J} D^{I}{ }_{J} \phi-\frac{5}{2 r^{2}} \phi^{2} \\
& +\frac{i}{2}\left(\psi_{I}\right)^{\dagger} \gamma^{m} \nabla_{m} \psi_{I}+\frac{1}{4 r}\left(\psi_{I}\right)^{\dagger}\left(\sigma^{3}\right)_{I}{ }^{J} \psi_{J} .
\end{aligned}
$$

Integrating out $D_{J}^{I}$ amounts to putting

$$
D_{J}^{I}=-\frac{i}{r}\left(\sigma^{3}\right)_{I}^{J} \phi
$$

The non-Abelian generalization can now also be easily identified from matching our result with [1].

We should stress that the correspondence between keeping a nonzero fermionic $\mathrm{KK}$ mode and turning on an $\mathrm{R}$-gauge field only works for the small tensor multiplet. We were not able to couple this multiplet to hypermultiplets in our approach. But of course the coupling to hypermultiplets can be obtained by turning on an R-gauge field along the time direction upon dimensional reduction. So that means that this correspondence is not one-to-one. Finally, there are many $5 \mathrm{~d}$ theories that are not obtained from the M5 brane such as those with hypermultiplets in other representations [1] whose UV completion may be five-dimensional rather than six-dimensional.

\section{NULL REDUCTION}

A general null reduction of the M5 brane was studied in [3]. Here we will stay with our example of $\mathbb{R} \times S^{5}$ with Lorentzian time along $\mathbb{R}$ for simplicity, although we believe that our results can be generalized to any Lorentzian sixmanifold without any new conceptional difficulties, beyond those we will address here. We will perform the dimensional reduction along the null direction that is formed out of the time direction and a circle fiber direction on $S^{5}$ when viewed as a circle fiber over $\mathbb{C} P^{2}$. However, once we specify a circle fiber, there are two null directions, $x^{+}$and $x^{-}$and we need to make a choice. We will make the choice such that we perform the dimensional reduction along the $x^{-}$direction. This choice of null direction is correlated with some chirality choices for the supersymmetry parameter that we wish to make, as we will now explain.

We start by writing the metric on $\mathbb{R} \times S^{5}$ as a metric over the base-manifold $\mathbb{R} \times \mathbb{C} P^{2}$. The M5 brane on (a Hopf circle bundle over) $\mathbb{R} \times \mathbb{C} P^{2}$ was first studied in [7]. We start by writing the $6 \mathrm{~d}$ metric in the form

$$
d s^{2}=r^{2}\left(d y+\kappa_{i} d x^{i}\right)^{2}-d t^{2}+H_{i j} d x^{i} d x^{j}
$$

where the five coordinates $x^{m}$ on $S^{5}$ are separated as $y \sim$ $y+2 \pi$ for the circle fiber, and $x^{i}$ for the base manifold $\mathbb{C} P^{2}$, and $\kappa_{i}$ is the graviphoton whose nonvanishing curvature components are

$$
w_{\hat{1} \hat{2}}=w_{\hat{3} \hat{4}}=\frac{2}{r^{2}}
$$

where the hats on these indices indicate that they are tangent space indices of $\mathbb{C} P^{2}$. Here we use $G_{i j}$ to denote the 4d metric tensor on $\mathbb{C} P^{2}$ whose inverse is denoted $G^{i j}$. Further details regarding this Hopf fibration over $\mathbb{C} P^{2}$ can be found in Appendix D.

We then also split the indices in the $5 \mathrm{~d}$ Killing spinor equation

$$
\nabla_{m} \mathcal{E}^{\alpha \dot{\alpha}}=\frac{i}{2 r}\left(\gamma_{m}\right)^{\alpha}{ }_{\beta} \mathcal{E}^{\beta \dot{\alpha}}
$$

on $S^{5}$ into two equations

$$
\nabla_{y} \mathcal{E}=\frac{i}{2 r} \gamma_{y} \mathcal{E}, \quad \nabla_{i} \mathcal{E}=\frac{i}{2 r} \gamma_{i} \mathcal{E}
$$

associated to the fiber and the base manifold respectively (and from now, we suppress the spinor indices). To analyze these equations further, we need expressions for these covariant derivatives in terms of spin connections and we need to express the $5 \mathrm{~d}$ gamma matrices and in terms of $4 \mathrm{~d}$ gamma matrices. To this end, we start by writing down expressions for the vielbein

$$
e^{\hat{t}}=d t, \quad e^{\hat{y}}=r\left(d y+\kappa_{i} d x^{i}\right), \quad e^{\hat{i}}=E_{j}^{\hat{i}} d x^{j},
$$

and its inverse

$$
e_{\hat{t}}=\partial_{t}, \quad e_{\hat{y}}=\frac{1}{r} \partial_{y}, \quad e_{\hat{i}}=E_{\hat{i}}^{j}\left(\partial_{j}-\kappa_{j} \partial_{y}\right) .
$$


Using these vielbeins, we may expand the $5 \mathrm{~d}$ gamma matrices $\gamma_{m}$ in terms of $4 \mathrm{~d}$ gamma matrices $\tilde{\gamma}_{i}=E_{i}^{\hat{i}_{i}} \gamma_{\hat{i}}$ and $\gamma:=\gamma^{\hat{1} \hat{2} \hat{3} \hat{4}}$ as follows:

$$
\gamma_{y}=r \gamma, \quad \gamma_{i}=\tilde{\gamma}_{i}+r \kappa_{i} \gamma,
$$

and then we use standard circle bundle expressions for the $5 \mathrm{~d}$ covariant derivative acting on a $5 \mathrm{~d}$ spinor $\psi$,

$$
\begin{aligned}
& \nabla_{y} \psi=\partial_{y} \psi-\frac{r^{2}}{8} w_{i j} \tilde{\gamma}^{i j} \psi \\
& \nabla_{i} \psi=\tilde{\nabla}_{i} \psi-\frac{r^{2}}{8} \kappa_{i} w_{k l} \tilde{\gamma}^{k l} \psi+\frac{r}{4} w_{i j} \tilde{\gamma}^{j} \gamma \psi
\end{aligned}
$$

where $\tilde{\nabla}_{i}$ denotes the covariant derivative with respect to the metric on the $4 \mathrm{~d}$ base space. We are now ready to express (3.1) in $4 d$ quantities,

$$
\begin{aligned}
\partial_{y} \mathcal{E}-\frac{r^{2}}{8} w_{i j} \gamma^{i j} \mathcal{E} & =\frac{i}{2} \gamma \mathcal{E}, \\
\nabla_{i} \mathcal{E}-\frac{r^{2}}{8} \kappa_{i} w_{k l} \gamma^{k l} \mathcal{E}+\frac{r}{4} w_{i j} \gamma^{j} \gamma \mathcal{E} & =\frac{i}{2 r}\left(\gamma_{i}+r \kappa_{i} \gamma\right) \mathcal{E},
\end{aligned}
$$

where now all quantities are $4 \mathrm{~d}$ quantities, and so we have dropped the tildes for notational simplicity. We may also express the second equation more simply as

$$
\mathcal{D}_{i} \mathcal{E}=\frac{i}{2 r} \gamma_{i} \mathcal{E}-\frac{r}{4} w_{i j} \gamma^{j} \gamma \mathcal{E}
$$

where we have introduced the curly derivative

$$
\mathcal{D}_{i} \psi=\nabla_{i} \psi-\kappa_{i} \partial_{y} \psi
$$

But let us first analyze the first equation. Plugging in the explicit form of $w_{i j}$, this equation reads

$$
\partial_{y} \mathcal{E}=\frac{1}{2}\left(\gamma^{\hat{1} \hat{2}}+\gamma^{\hat{3} \hat{4}}+i \gamma\right) \mathcal{E} .
$$

Of course the spinor $\mathcal{E}^{\alpha \dot{\alpha}}$ has four different indices $\alpha$. To see the meaning of these various indices more clearly, we will introduce a spin notation $\alpha=\left(s_{1}, s_{2}\right)$ where the spins $s_{1}$ and $s_{2}$ are defined by

$$
\frac{i}{2} \gamma^{\hat{1} \hat{2} \mathcal{E}}=s_{1} \mathcal{E}, \quad \frac{i}{2} \gamma^{\hat{3} \hat{4}} \mathcal{E}=s_{2} \mathcal{E} .
$$

Let us first consider the spinor component $\left(s_{1}, s_{2}\right)=$ $(+,+)$ where \pm represent spins $\pm \frac{1}{2}$. The Killing spinor equations then reduce to

$$
\partial_{y} \mathcal{E}=-\frac{3 i}{2} \mathcal{E}, \quad \mathcal{D}_{i} \mathcal{E}=0
$$

Moving up to 6d, we have the conformal Killing spinor solution

$$
\varepsilon=e^{\frac{i}{2 r} t-\frac{3 i}{2} y} \mathcal{E}+e^{-\frac{i}{2 r} t+\frac{3 i}{2} y} \mathcal{F} .
$$

This is the singlet solution. The other cases are $\left(s_{1}, s_{2}\right)=$ $\{(-,-),(+,-),(-,+)\}$ that form a triplet. For any of these components, the first Killing spinor equation becomes

$$
\partial_{y} \mathcal{E}=\frac{i}{2} \mathcal{E}
$$

and then the $6 \mathrm{~d}$ solution becomes

$$
\varepsilon=e^{\frac{i}{2 r} t+\frac{i}{2} y} \mathcal{E}+e^{-\frac{i}{2 r} t-\frac{i}{2} y} \mathcal{F},
$$

but the Killing spinor equations for $\mathcal{E}$ and $\mathcal{F}$ now become more complicated. We introduce light-cone coordinates

$$
x^{ \pm}=\frac{1}{\sqrt{2}}(t \pm r y) .
$$

Expressed in these light-cone coordinates, the singlet solution is

$$
\varepsilon=e^{\frac{i}{r \sqrt{2}}\left(-x^{+}+2 x^{-}\right)} \mathcal{E}+e^{-\frac{i}{r \sqrt{2}}\left(-x^{+}+2 x^{-}\right)} \mathcal{F}
$$

and the triplet solutions are

$$
\varepsilon=e^{\frac{i}{r \sqrt{2}} x^{+}} \mathcal{E}+e^{-\frac{i}{r \sqrt{2}} x^{+}} \mathcal{F}
$$

Since these triplet supersymmetry parameters do not depend on $x^{-}$, the corresponding supersymmetry survives upon dimensional reduction along $x^{-}$without any need to turn on an R-gauge field. While this is nice, the price we have to pay is having a more complicated Killing spinor equation.

We will study the singlet solution instead. This has a simpler Killing spinor equation, and it gives us an opportunity to study a situation where the supersymmetry parameter depends nontrivially on the fiber direction along which we dimensionally reduce. But again the question arises, along which direction we shall reduce. Let us start by recalling the $6 \mathrm{~d}$ Weyl condition $\Gamma \varepsilon=-\varepsilon$ that we will write as

$$
\Gamma^{t y} \Gamma^{1234} \varepsilon=-\varepsilon
$$

As we mentioned in the Introduction, we also want to impose the Weyl projection

$$
\Gamma_{M} \varepsilon v^{M}=0
$$

where $v^{M}$ is now to be either one of the light-cone directions, $v^{M}=\delta_{ \pm}^{M}$. So the above Weyl projection amounts to 


$$
\Gamma_{ \pm} \varepsilon=0
$$

where

$$
\Gamma_{ \pm}=\frac{1}{\sqrt{2}}\left(\Gamma_{t} \pm \frac{1}{r} \Gamma_{y}\right)
$$

so we may also express this Weyl projection as

$$
\Gamma^{t y} \varepsilon=\mp \varepsilon \text {. }
$$

Now by combining (3.2) and (3.3), we get

$$
\Gamma^{1234} \varepsilon= \pm \varepsilon .
$$

The singlet supersymmetry parameter has $\Gamma^{1234} \varepsilon=-\varepsilon$ and therefore we shall take $v^{M}=\delta_{-}^{M}$ and perform the dimensional reduction along the $x^{-}$direction. Let us write down the singlet solution again as

$$
\varepsilon=e^{\frac{i \sqrt{2}}{r} x^{-}} \mathcal{E}+e^{-\frac{i \sqrt{2}}{r} x^{-}} \mathcal{F} .
$$

Then upon dimensional reduction, we shall expand the fermionic field in the same modes as

$$
\psi=e^{\frac{i \sqrt{2}}{r} x^{-}} \chi+e^{-\frac{i \sqrt{2}}{r} x^{-}} \zeta .
$$

Of course we do not know the non-Abelian supersymmetry variations for the M5 brane. The strategy therefore will be to start with the Abelian supersymmetry variations for the M5 brane, and reduce these along the $x^{-}$direction by using the above mode expansion for the fermionic field. These steps are in parallel with what we have already done when we reduced along the time direction, although the reduction along $x^{-}$requires a lot more computations. Once we have obtained these Abelian supersymmetries and Lagrangian, the generalization to the non-Abelian case will be examined. We start by replacing derivatives with gauge covariant derivatives and examine the term in the variation of the Lagrangian that is cubic in the fermionic field. But this term is vanishing, not because of some Fierz rearrangment, but simply because, as we will see, the supersymmetry variation of the following combination of gauge fields is vanishing, ${ }^{2}$

$$
\delta\left(A_{i}-\kappa_{i} A_{y}\right)=0
$$

and it is precisely this combination that enters in the kinetic term for the fermionic field

$$
i \chi^{\dagger} \gamma^{i} \mathcal{D}_{i} \chi
$$

\footnotetext{
${ }^{2}$ In $6 \mathrm{~d}$ we also have the gauge fixing condition $A_{M} v^{M}=A_{-}=0$ that can be seen as a consequence of $A_{M}=B_{M N} v^{N}$.
}

So when we vary the gauge potential in this term, there will be no cubic term generated. Let us now show this in more detail. Let us start with the $6 \mathrm{~d}$ supersymmetry variation

$$
\delta A_{M}=-i \bar{\psi} \Gamma_{M N} \varepsilon v^{N}
$$

from which we obtain

$$
\delta A_{i}=-\frac{i r}{\sqrt{2}} \kappa_{i} \bar{\psi} \varepsilon, \quad \delta A_{+}=-i \bar{\psi} \varepsilon .
$$

Then

$$
\mathcal{D}_{i} \psi=\left(D_{i}-\kappa_{i} D_{y}\right) \psi=\left(D_{i}-\frac{r}{\sqrt{2}} \kappa_{i} D_{+}\right) \psi
$$

The important observation is now that

$$
\delta \mathcal{D}_{i}=-i e \delta\left(A_{i}-\frac{r}{\sqrt{2}} \kappa_{i} A_{+}\right)=0
$$

For this computation we have used

$$
\Gamma_{ \pm}=\Gamma_{\hat{\underline{1}}}, \quad \Gamma_{i}=\tilde{\Gamma}_{i}+\frac{r}{\sqrt{2}}\left(\Gamma_{+}-\Gamma_{-}\right),
$$

and then

$$
\Gamma_{i \pm}=\tilde{\Gamma}_{i} \Gamma_{ \pm}+\frac{r}{\sqrt{2}} \kappa_{i} \Gamma_{+-}
$$

We have

$$
\Gamma_{ \pm}=\frac{1}{\sqrt{2}}\left(\Gamma^{t} \pm \frac{1}{r} \Gamma_{y}\right), \quad \Gamma^{ \pm}=\frac{1}{\sqrt{2}}\left(\Gamma^{t} \pm r \Gamma^{y}\right),
$$

and then we get

$$
\Gamma_{+-}=\Gamma^{\hat{\imath} \hat{y}}
$$

We impose the Weyl projection

$$
\Gamma_{-} \varepsilon=0
$$

and then we get

$$
\Gamma_{-} \Gamma_{+} \varepsilon=\left(\left\{\Gamma_{-}, \Gamma_{+}\right\}-\Gamma_{+} \Gamma_{-}\right) \varepsilon=-2 \varepsilon,
$$

where we notice the metric is

$$
d s^{2}=-2 e^{\hat{+}} e^{\hat{\imath}}+e^{\hat{i}} e^{\hat{i}} .
$$

Now having shown that $\delta \mathcal{D}_{i}=0$ is, as we will see below, just one crucial step among many other steps towards obtaining a supersymmetry non-Abelian Lagrangian. 
We begin with assuming the gauge group is Abelian and first study the supersymmetry variation of the tensor gauge field in $6 \mathrm{~d}$,

$$
\delta H_{M N P}=-3 i \partial_{M}\left(\bar{\psi} \Gamma_{N P} \varepsilon\right)
$$

for an anticommuting supersymmetry parameter, for which we have the relation

$$
\begin{aligned}
\bar{\varepsilon} \Gamma_{M N} \psi & =\left(\varepsilon^{T} C \Gamma_{M N} \psi\right)^{T}=-\psi^{T}\left(-C \Gamma_{M N} C^{-1}\right)(-C) \varepsilon \\
& =-\bar{\psi} \Gamma_{M N} \varepsilon
\end{aligned}
$$

where we used the 11d Majorana condition. We would first like to show a correspondence with the fermionic equation of motion and self-duality of $H_{M N P}$. In 6d, this correspondence is almost trivial to show. Namely, we have

$$
\left(\delta H_{M N P}\right)^{-}=-\frac{i}{2} \nabla_{Q}\left(\bar{\psi} \Gamma^{Q} \Gamma_{M N P} \varepsilon\right)
$$

and by using the identity $\Gamma^{Q} \Gamma_{M N P} \Gamma_{Q}=0$ and $\nabla_{M} \varepsilon=\Gamma_{M} \eta$, we get

$$
\left(\delta H_{M N P}\right)^{-}=-\frac{i}{2} \nabla_{Q} \bar{\psi} \Gamma^{Q} \Gamma_{M N P} \varepsilon
$$

and we see that this variation vanishes on the fermionic equation of motion $\Gamma^{M} \nabla_{M} \psi=0$.

We would now like to show this correspondence between self-duality and the fermionic equation of motion again, but now in light-cone coordinates, following closely [3]. To this end, we define

$$
\mathcal{G}_{i j}=G_{i j}-r \sqrt{2} F_{i+} \kappa_{j}
$$

where

$$
G_{i j}=H_{i j+}, \quad F_{i+}=H_{i+-},
$$

and we want to show that the self-dual part vanishes, $\left(\delta \mathcal{G}_{i j}\right)^{+}=0$, on the fermionic equation of motion. So we first need to obtain the explicit expressions for the supersymmetry variation and for the fermionic equation of motion in light-cone coordinates. We begin with the supersymmetry variation. We have

$$
\begin{aligned}
\delta G_{i j} & =-2 i \nabla_{i}\left(\bar{\psi} \Gamma_{j+} \varepsilon\right)-i \partial_{+}\left(\bar{\psi} \Gamma_{i j} \varepsilon\right), \\
\delta F_{i+} & =-i \partial_{i}\left(\bar{\psi} \Gamma_{+-} \varepsilon\right)+i \partial_{+}\left(\bar{\psi} \Gamma_{i-} \varepsilon\right)-i \partial_{-}\left(\bar{\psi} \Gamma_{i+} \varepsilon\right),
\end{aligned}
$$

where $\nabla_{i}$ are $4 \mathrm{~d}$ covariant derivatives. We expand

$$
\mathcal{E}=e^{\frac{i \sqrt{2}}{r} x^{-}} \mathcal{E}+e^{-\frac{i \sqrt{2}}{r} x^{-}} \mathcal{F}, \quad \psi=e^{\frac{i \sqrt{2}}{r} x^{-}} \chi+e^{-\frac{i \sqrt{2}}{r} x^{-}} \zeta,
$$

where

$$
\nabla_{i} \mathcal{E}=-\frac{3 i}{2} \kappa_{i} \mathcal{E}, \quad \partial_{+} \mathcal{E}=-\frac{i}{r \sqrt{2}} \mathcal{E},
$$

and corresponding relations for $\mathcal{F}$. We also expand

$$
\begin{aligned}
\Gamma_{i \pm} & =\tilde{\Gamma}_{i} \Gamma_{ \pm}+\frac{r}{\sqrt{2}} \kappa_{i} \Gamma_{+-}, \\
\Gamma_{i j} & =\tilde{\Gamma}_{i j}-r \sqrt{2} \kappa_{i} \tilde{\Gamma}_{j}\left(\Gamma_{+}-\Gamma_{-}\right) .
\end{aligned}
$$

Then we get

$$
\begin{aligned}
\delta G_{i j}= & -2 i \nabla_{i}\left(\bar{\chi} \tilde{\Gamma}_{j} \Gamma_{+} \mathcal{E}\right)-i \sqrt{2} r \nabla_{i}\left(\bar{\chi} \Gamma_{+-} \mathcal{E} \kappa_{j}\right) \\
& -i \partial_{+}\left(\bar{\chi} \tilde{\Gamma}_{i j} \mathcal{E}\right)+i \sqrt{2} r \partial_{+}\left(\kappa_{i} \bar{\chi} \tilde{\Gamma}_{j} \Gamma_{+} \mathcal{E}\right) .
\end{aligned}
$$

We may now notice the appearance of a curly derivative from

$$
-2 i\left(\nabla_{i}-\frac{r}{\sqrt{2}} \kappa_{i} \partial_{+}\right)\left(\bar{\chi} \tilde{\Gamma}_{j} \Gamma_{+} \mathcal{E}\right)=-2 i \mathcal{D}_{i}\left(\bar{\chi} \tilde{\Gamma}_{j} \Gamma_{+} \mathcal{E}\right),
$$

where we assume that $\partial_{+} \kappa_{i}=0$. So then we have

$\delta G_{i j}=-2 i \mathcal{D}_{i}\left(\bar{\chi} \tilde{\Gamma}_{j} \Gamma_{+} \mathcal{E}\right)-i \sqrt{2} r \nabla_{i}\left(\bar{\chi} \Gamma_{+-} \mathcal{E} \kappa_{j}\right)-i \partial_{+}\left(\bar{\chi} \tilde{\Gamma}_{i j} \mathcal{E}\right)$

We have

$$
\delta F_{i+}=-i \nabla_{i}\left(\bar{\chi} \Gamma_{+-} \mathcal{E}\right)+\frac{i r}{\sqrt{2}} \kappa_{i} \partial_{+}\left(\bar{\chi} \Gamma_{+-} \mathcal{E}\right)
$$

and then we get

$$
\delta \mathcal{G}_{i j}=-2 i \mathcal{D}_{i}\left(\bar{\chi} \tilde{\Gamma}_{j} \Gamma_{+} \mathcal{E}\right)-i \sqrt{2} r \bar{\chi} \Gamma_{+-} \mathcal{E} \nabla_{i} \kappa_{j}-i \partial_{+}\left(\bar{\chi} \tilde{\Gamma}_{i j} \mathcal{E}\right)
$$

or if we define

$$
w_{i j}=\nabla_{i} \kappa_{j}-\nabla_{j} \kappa_{i}
$$

then we can write this as

$$
\delta \mathcal{G}_{i j}=-2 i \mathcal{D}_{i}\left(\bar{\chi} \tilde{\Gamma}_{j} \Gamma_{+} \mathcal{E}\right)-\frac{i r}{\sqrt{2}} \bar{\chi} \Gamma_{+-} \mathcal{E} w_{i j}-i \partial_{+}\left(\bar{\chi} \tilde{\Gamma}_{i j} \mathcal{E}\right) .
$$

We are now interested in extracting the self-dual part of this variation. To do this, we first recall the Weyl projection

$$
\Gamma_{-} \mathcal{E}=0 \text {. }
$$

We have

$$
\Gamma_{ \pm}=\frac{1}{\sqrt{2}}\left(\Gamma_{t} \pm \frac{1}{r} \Gamma_{y}\right), \quad \Gamma^{ \pm}=\frac{1}{\sqrt{2}}\left(\Gamma^{t} \pm r \Gamma^{y}\right) .
$$

The Weyl projection can be written in the following alternative forms: 


$$
\Gamma^{\hat{\hat{y}} \hat{\mathcal{E}}}=\mathcal{E}, \quad \Gamma_{+-} \mathcal{E}=\mathcal{E} .
$$

Expressed in terms of $4 \mathrm{~d}$ gamma matrices, we get

$$
\delta \mathcal{G}_{i j}=2 \sqrt{2} i \mathcal{D}_{i} \chi^{*} \gamma_{j} \mathcal{E}-\frac{i r}{\sqrt{2}} \chi^{*} \mathcal{E} w_{i j}-i \partial_{+}\left(\chi^{*} \gamma_{i j} \mathcal{E}\right) .
$$

We can further write this as

$$
\begin{aligned}
\delta \mathcal{G}_{i j}= & \frac{i}{\sqrt{2}} \mathcal{D}_{k} \chi^{*}\left[\gamma^{k}, \gamma_{i j}\right] \mathcal{E}-i \partial_{+} \chi^{*} \gamma_{i j} \mathcal{E} \\
& -\frac{1}{r \sqrt{2}} \chi^{*} \gamma_{i j} \mathcal{E}-\frac{i r}{\sqrt{2}} \chi^{*} \mathcal{E} w_{i j} .
\end{aligned}
$$

Here we have rewritten this in terms of $6 \mathrm{~d}$ Weyl components so that now all that remains of the $\Gamma_{-} \mathcal{E}=0$ Weyl projection is

$$
\gamma \mathcal{E}=-\mathcal{E}
$$

which amounts to that $\gamma_{i j} \mathcal{E}$ will be self-dual, and also $\gamma_{i j} \gamma_{k} \mathcal{E}$ will be antiself-dual simply because $\gamma_{k} \mathcal{E}$ is satisfying the opposite Weyl projection

$$
\gamma \gamma_{k} \mathcal{E}=\gamma_{k} \mathcal{E}
$$

as $\left\{\gamma_{k}, \gamma\right\}=0$. Also since $w_{i j}$ is self-dual, we can now extract the self-dual part of the variation,

$$
\begin{aligned}
\left(\delta \mathcal{G}_{i j}\right)^{+}= & \frac{i}{\sqrt{2}} \mathcal{D}_{k} \chi^{*} \gamma^{k} \gamma_{i j} \mathcal{E}-i \partial_{+} \chi^{*} \gamma_{i j} \mathcal{E} \\
& -\frac{1}{r \sqrt{2}} \chi^{*} \gamma_{i j} \mathcal{E}-\frac{i r}{\sqrt{2}} \chi^{*} \mathcal{E} w_{i j} .
\end{aligned}
$$

We can also write this in the form

$$
\delta \mathcal{G}_{i j}=-2 \sqrt{2} i \mathcal{F}^{*} \gamma_{j} \mathcal{D}_{i} \zeta+\frac{i r}{\sqrt{2}} \mathcal{F}^{*} \zeta w_{i j}+i \partial_{+}\left(\mathcal{F}^{*} \gamma_{i j} \zeta\right)
$$

and then

$$
\begin{aligned}
\left(\delta \mathcal{G}_{i j}\right)^{+}= & -\frac{i}{\sqrt{2}} \mathcal{F}^{*} \gamma_{i j} \gamma^{k} \mathcal{D}_{k} \zeta+\frac{i r}{\sqrt{2}} \mathcal{F}^{*} \zeta w_{i j}+i \mathcal{F}^{*} \gamma_{i j} \partial_{+} \zeta \\
& +\frac{1}{r \sqrt{2}} \mathcal{F}^{*} \gamma_{i j} \zeta
\end{aligned}
$$

that we can write as

$$
=-\frac{i}{\sqrt{2}} \mathcal{F}^{*} \gamma_{i j}\left(\gamma^{k} \mathcal{D}_{k} \zeta-\sqrt{2} \partial_{+} \zeta+\frac{i}{r} \zeta\right)+\frac{i r}{\sqrt{2}} \mathcal{F}^{*} \zeta w_{i j}
$$

We now use the identity

$$
\mathcal{E}=\frac{i r^{2}}{8} \gamma^{i j} \mathcal{E} w_{i j}
$$

to rewrite one term as

$$
-\frac{1}{r \sqrt{2}} \chi^{*} \gamma_{i j} \mathcal{E}=-\frac{i r}{8 \sqrt{2}} \chi^{*} \gamma_{i j} \gamma^{k l} \mathcal{E} w_{k l}
$$

and then we decompose

$$
\gamma_{i j} \gamma^{k l}=\left\{\gamma_{i j}, \gamma^{k l}\right\}-\gamma^{k l} \gamma_{i j} .
$$

Noting that $\left\{\gamma_{i j}, \gamma^{k l}\right\}=-8 \delta_{i j}^{k l}$ when acting on self-dual $w_{k l}$, the first term gives rise to a term

$$
-\frac{i r}{\sqrt{2}} \chi^{*} \mathcal{E} w_{i j}
$$

that cancels that corresponding term in $\delta \mathcal{G}_{i j}$, and we are left with

$$
\delta \mathcal{G}_{i j}=\frac{i}{\sqrt{2}} \mathcal{D}_{k} \chi^{*}\left[\gamma^{k}, \gamma_{i j}\right] \mathcal{E}-i \partial_{+} \chi^{*} \gamma_{i j} \mathcal{E}+\frac{i r}{8 \sqrt{2}} \chi^{*} \gamma^{k l} \gamma_{i j} \mathcal{E}
$$

and consequently

$$
\left(\delta \mathcal{G}_{i j}\right)^{+}=\frac{i}{\sqrt{2}} \mathcal{D}_{k} \chi^{*} \gamma^{k} \gamma_{i j} \mathcal{E}-i \partial_{+} \chi^{*} \gamma_{i j} \mathcal{E}+\frac{i r}{8 \sqrt{2}} \chi^{*} \gamma^{k l} \gamma_{i j} \mathcal{E}
$$

We can write this as

$$
\left(\delta \mathcal{G}_{i j}\right)^{+}=\frac{i}{\sqrt{2}}\left(\mathcal{D}_{k} \chi^{*} \gamma^{k}-\sqrt{2} \partial_{+} \chi^{*}+\frac{r}{8} \chi^{*} \gamma^{k l} w_{k l}\right) \gamma_{i j} \mathcal{E} .
$$

We now wish to show that this vanishes when the fermionic equation of motion is satisfied. Taking the complex conjugate of what is inside the parentheses, we get the requirement

$$
\gamma^{i} \mathcal{D}_{i} \chi-\sqrt{2} \partial_{+} \chi-\frac{r}{8} \gamma^{k l} \chi w_{k l}=0
$$

and indeed this is (a Weyl component of) the equation of motion.

Let us complete the supersymmetry variations. We have

$$
\begin{aligned}
\delta F_{i+} & =-i\left(\nabla_{i}(\bar{\chi} \mathcal{E})-\frac{r}{\sqrt{2}} \kappa_{i} \partial_{+}(\bar{\chi} \mathcal{E})\right) \\
& =-i \mathcal{D}_{i}(\bar{\chi} \mathcal{E}) \\
& =-i \mathcal{D}_{i}\left(\chi^{*} \mathcal{E}\right)
\end{aligned}
$$

and, quite interestingly,

$$
\delta \mathcal{F}_{i j}=-\frac{i r}{\sqrt{2}} \chi^{*} \mathcal{E} w_{i j} .
$$


This is interesting, because it is zero, up to a term that is proportional to $w_{i j}$. This is nothing like the usual supersymmetry variation, and in fact $\delta \mathcal{D}_{i}=0$. And trivially

$$
\left(\delta \mathcal{F}_{i j}\right)^{-}=0
$$

since $w_{i j}$ is self-dual. We do not even need to use the fermionic equation of motion here.

We will now derive a 5d Lagrangian from the self-dual tensor field in $6 \mathrm{~d}$ dimensions, following closely [3]. We start by noting that

$$
\begin{aligned}
H_{\hat{i} \hat{j} \hat{\jmath}} & =E_{\hat{i}}^{i} E_{\hat{j}}^{j}\left(H_{i j \pm}-r \sqrt{2} H_{i+-} \kappa_{j}\right), \\
H_{\hat{i} \hat{j} \hat{k}} & =E_{\hat{i}}^{i} E_{\hat{j}}^{j} E_{\hat{k}}^{k}\left(H_{i j k}-\frac{3 r}{\sqrt{2}} H_{i j+} \kappa_{k}+\frac{3 r}{\sqrt{2}} H_{i j-} \kappa_{k}\right), \\
H_{\hat{i} \hat{\dagger} \hat{\imath}} & =E_{\hat{i}}^{i} H_{i+-},
\end{aligned}
$$

or if we define

$$
F_{i j}=H_{i j-}, \quad G_{i j}=H_{i j+}, \quad F_{i+}=H_{i+-},
$$

then

$$
\begin{aligned}
H_{\hat{i} \hat{j} \hat{+}} & =E_{\hat{i}}^{i} E_{\hat{j}}^{j}\left(G_{i j}-r \sqrt{2} F_{i+} \kappa_{j}\right), \\
H_{\hat{i} \hat{j} \hat{\imath}} & =E_{\hat{i}}^{i} E_{\hat{j}}^{j}\left(F_{i j}-r \sqrt{2} F_{i+} \kappa_{j}\right), \\
H_{\hat{i} \hat{j} \hat{k}} & =E_{\hat{i}}^{i} E_{\hat{j}}^{j} E_{\hat{k}}^{k}\left(H_{i j k}+\frac{3 r}{\sqrt{2}}\left(F_{i j}-G_{i j}\right) \kappa_{k}\right), \\
H_{\hat{i} \hat{+} \hat{\imath}} & =E_{\hat{i}}^{i} F_{i+} .
\end{aligned}
$$

We have the Bianchi identity

$$
3 \partial_{[i} H_{j k]+}-\partial_{+} H_{i j k}=0
$$

and we have the self-duality relation

$$
H_{\hat{i} \hat{j} \hat{k}}=\varepsilon_{\hat{i} \hat{j} \hat{k}}^{\hat{l} \hat{+} \hat{-}} H_{\hat{l} \hat{+} \hat{*}} .
$$

We define

$$
\varepsilon_{\hat{i} \hat{j} \hat{k} \hat{l} \hat{+} \hat{-}}=\varepsilon_{\hat{i} \hat{j} \hat{k} \hat{l}}
$$

so we have

$$
H_{\hat{i} \hat{j} \hat{k}}=-\varepsilon_{\hat{i} \hat{j} \hat{k}}^{\hat{l}} H_{\hat{l} \hat{+} \wedge}
$$

that we can write as

$$
H_{i j k}+\frac{3 r}{\sqrt{2}}\left(F_{i j}-G_{i j}\right) \kappa_{k}+\varepsilon_{i j k}^{l} F_{l+}=0 .
$$

The Bianchi identity (3.4) then becomes

$$
3 \partial_{[i} G_{j k]}=-\partial_{+}\left(\frac{3 r}{\sqrt{2}}\left(F_{i j}-G_{i j}\right) \kappa_{k}+\varepsilon_{i j k}^{l} F_{l+}\right) .
$$

We define

$$
\mathcal{G}_{i j}=G_{i j}-r \sqrt{2} F_{i+} \kappa_{j}, \quad \mathcal{F}_{i j}=F_{i j}-r \sqrt{2} F_{i+} \kappa_{j},
$$

that enable us to express (3.5) in the following simple form:

$$
\varepsilon^{i j k l} \mathcal{D}_{i} \mathcal{G}_{j k}=-2 \partial_{+} F_{+}^{l}
$$

and from

$$
H_{\hat{i} \hat{j} \hat{+}}=\frac{1}{2} \varepsilon_{\hat{i} \hat{j} \hat{+}} \hat{k} \hat{l} \hat{+} H_{\hat{k} \hat{l} \hat{+}}
$$

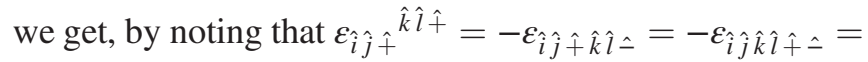
$-\varepsilon_{\hat{i} \hat{j} \hat{k} \hat{l}}$,

$$
\mathcal{G}_{i j}=-\frac{1}{2} \varepsilon_{i j}{ }^{k l} \mathcal{G}_{k l}, \quad \mathcal{F}_{i j}=\frac{1}{2} \varepsilon_{i j}{ }^{k l} \mathcal{F}_{k l} .
$$

The next step will therefore be to replace straight capital letters with curly ones,

$$
\begin{aligned}
& \partial_{[i}\left(\mathcal{G}_{j k]}+r \sqrt{2} F_{j+} \kappa_{k}\right) \\
& \quad+\partial_{+}\left(\frac{r}{\sqrt{2}}\left(\mathcal{F}_{i j}-\mathcal{G}_{i j}\right) \kappa_{k}+\frac{1}{3} \varepsilon_{i j k}^{l} F_{l+}\right)=0
\end{aligned}
$$

because then we can dualize and get

$$
\begin{aligned}
- & D_{i} \mathcal{G}^{i l}+\frac{r}{\sqrt{2}} \varepsilon^{i j k l} D_{i}\left(F_{j+} \kappa_{k}\right) \\
& +\frac{r}{\sqrt{2}} \kappa_{i} \partial_{+}\left(\mathcal{F}^{i l}+\mathcal{G}^{i l}\right)+\partial_{+} F_{+}^{l}=0 .
\end{aligned}
$$

As a consequence of this equation, we have

$$
-\left(D_{i} \mathcal{G}^{i l}\right) \kappa_{l}+\partial_{+} F^{l} \kappa_{l}=0
$$

that we can also write as

$$
-D_{i}\left(\mathcal{G}^{i j} \kappa_{j}\right)+\frac{1}{2} G^{i l} w_{i l}+\partial_{+} F_{+}^{l} \kappa_{l}=0
$$

but the second term is vanishing, as one can see by replacing $G^{i l}$ with $\mathcal{G}^{i l}$ which is antiself-dual so contracting with a self-dual $w_{i l}$ gives zero. And moreover $\kappa^{l} w_{i l}=0$. So we have

$$
D_{i}\left(\mathcal{G}^{i j} \kappa_{j}\right)=\kappa_{i} \partial_{+} F^{i}+
$$

which will be a useful relation that we will use later. We may also write 
$-\mathcal{D}_{i} \mathcal{G}^{i l}+\frac{r}{\sqrt{2}} \varepsilon^{i j k l} D_{i}\left(F_{j+} \kappa_{k}\right)+\frac{r}{\sqrt{2}} \kappa_{i} \partial_{+} \mathcal{F}^{i l}+\partial_{+} F_{+}^{l}=0$

We have the Bianchi identity

$$
3 \partial_{[i} H_{j k]-}=\partial_{-} H_{i j k}
$$

but if we put $\partial_{-}=0$ upon dimensional reduction, then this reduces to

$$
\varepsilon^{i j k l} \partial_{i} F_{j k}=0
$$

Again replacing straight capital $F$ with curly $\mathcal{F}$, we first get

$$
\varepsilon^{i j k l} \partial_{i}\left(\mathcal{F}_{j k}+r \sqrt{2} F_{j+} \kappa_{k}\right)=0
$$

and then by using self-duality this becomes

$$
D_{i} \mathcal{F}^{i l}+\frac{r}{\sqrt{2}} \varepsilon^{i j k l} D_{i}\left(F_{j+} \kappa_{k}\right)=0 .
$$

But the nicest way to express this same equation is as

$$
\varepsilon^{i j k l} \mathcal{D}_{i} \mathcal{F}_{j k}=0
$$

We have the Bianchi identity

$$
2 \partial_{[i} F_{j]+}+\partial_{+} F_{i j}=0
$$

Replacing $F$ with $\mathcal{F}$ it becomes

$$
2 \mathcal{D}_{[i} F_{j]+}+\partial_{+} \mathcal{F}_{i j}=0
$$

Finally, we return to

$$
H_{i j k}+\frac{3 r}{\sqrt{2}}\left(F_{i j}-G_{i j}\right) \kappa_{k}+\varepsilon_{i j k}^{l} F_{l+}=0
$$

and apply the Bianchi identity $\varepsilon^{i j k l} \partial_{l} H_{i j k}=0$. We then get

$$
\mathcal{D}^{i} F_{i+}=\frac{r}{2 \sqrt{2}} \mathcal{F}_{i j} w^{i j}
$$

where we define

$$
\mathcal{D}_{i}=D_{i}-\frac{r}{\sqrt{2}} \kappa_{i} \partial_{+}
$$

We thus have two types of equations of motion,

$$
\begin{aligned}
-\mathcal{D}_{i} \mathcal{G}^{i l}+\frac{r}{\sqrt{2}} \varepsilon^{i j k l} D_{i}\left(F_{j+} \kappa_{k}\right)+\frac{r}{\sqrt{2}} \kappa_{i} \partial_{+} \mathcal{F}^{i l}+\frac{1}{2} \partial_{+} F^{l}{ }_{+} & =0, \\
\mathcal{D}^{i} F_{i+}-\frac{r}{2 \sqrt{2}} \mathcal{F}_{i j} w^{i j} & =0,
\end{aligned}
$$

and in addition to these, we have the self-duality equations

$$
\mathcal{G}_{i j}=-\frac{1}{2} \varepsilon_{i j}{ }^{k l} \mathcal{G}_{k l}, \quad \mathcal{F}_{i j}=\frac{1}{2} \varepsilon_{i j}{ }^{k l} \mathcal{F}_{k l} \text {. }
$$

The equation

$$
D_{i} \mathcal{F}^{i l}+\frac{r}{\sqrt{2}} \varepsilon^{i j k l} D_{i}\left(F_{j+} \kappa_{k}\right)=0
$$

surely looks very much like an independent equation of motion, but actually it is not. It is a direct consequence of $\varepsilon^{i j k l} \partial_{i} F_{j k}=0$ together with the self-duality equation of motion for $\mathcal{F}_{i j}$. That means we do not need to demand that Eq. (3.6) follows from an action upon the variation of a gauge field as one normally would expect. Now one may ask some questions about the number of components. Let us be very brief and just notice that self-dual $H_{M N P}$ has 10 components, just as do self-dual $F_{i j}$ and $F_{i+}$ together, as $6+4=10$. So we do not expect $G_{i j}$ shall be part of the supermultiplet upon dimensional reduction. Only $F_{i j}, F_{i+}$ should be part of the vector muliplet. It then seems reasonable to assume that the antiself-dual $\mathcal{G}_{i j}$ shall be viewed as a Lagrange multiplier field that is imposing self-duality on $\mathcal{F}_{i j}$, rather than as a dynamical field that contributes to additional degrees of freedom. We now make the following ansatz for a gauge field Lagrangian:

$\mathcal{L}_{A}=b \mathcal{F}^{i j} \mathcal{G}_{i j}+c F^{i}{ }_{+} F_{i+}+d \varepsilon^{i j k l} F_{i j} F_{k+} \kappa_{l}+e \varepsilon^{i j k l} G_{i j} F_{k+} \kappa_{l}$

and treat $\mathcal{G}_{i j}$ (assumed to be antiself-dual from the outset), $A_{i}$, and $A_{+}$as independent fields that we shall vary to derive the classical equations of motion. Then these equations of motion become

$$
\begin{array}{r}
\mathcal{F}_{i j}-\frac{1}{2} \varepsilon_{i j}{ }^{k l} \mathcal{F}_{k l}=0, \\
(b r \sqrt{2}+2 e) D_{i}\left(\mathcal{G}^{i j} \kappa_{j}\right)-2 c D^{i} F_{i+}-d \mathcal{F}_{i j} w^{i j}=0, \\
-2 b D_{i} \mathcal{G}^{i m}+(b r \sqrt{2}+2 e) \kappa_{i} \partial_{+} \mathcal{G}^{i m}+2 d \varepsilon^{m i k l} D_{i}\left(F_{k+} \kappa_{l}\right) \\
+2 d \partial_{+} \mathcal{F}^{m j} \kappa_{j}+2 c \partial_{+} F^{m}{ }_{+}=0 .
\end{array}
$$

We now write the second relation as

$$
-2 c D_{i} F_{+}^{i}+(b r \sqrt{2}+2 e) \kappa_{i} \partial_{+} F_{+}^{i}-d \mathcal{F}_{i j} w^{i j}=0 .
$$

By now requiring the combination $\mathcal{D}_{i}=D_{i}-\frac{r}{\sqrt{2}} \kappa_{i} \partial_{+}$to appear, we get the following equations:

$$
\begin{array}{rlrl}
\frac{b r \sqrt{2}+2 e}{2 c} & =\frac{r}{\sqrt{2}}, & \frac{b r \sqrt{2}+2 e}{2 b}=\frac{r}{\sqrt{2}}, \\
\frac{d}{b} & =-\frac{r}{\sqrt{2}}, \quad \frac{c}{b}=1 .
\end{array}
$$


These equations have the following unique solution:

$$
\begin{aligned}
& b=1, \quad c=1, \\
& d=-\frac{r}{\sqrt{2}}, \quad e=0
\end{aligned}
$$

up to one overall constant. Fixing that overall constant to be $1 / 4$, the Lagrangian is given by

$$
\mathcal{L}_{A}=\frac{1}{4}\left(\mathcal{F}^{i j} \mathcal{G}_{i j}+F^{i}{ }_{+} F_{i+}-\frac{r}{\sqrt{2}} \varepsilon^{i j k l} \mathcal{F}_{i j} F_{k+} \kappa_{l}\right)
$$

where we have also replaced $F$ with $\mathcal{F}$ in the graviphoton term, which we can do freely by just noting that $\kappa_{j} \kappa_{l}=0$ upon antisymmetrization in $j$ and $l$. The supersymmetry variation of this Lagrangian is

$$
\begin{aligned}
\delta \mathcal{L}_{A}= & -\frac{i}{\sqrt{2}} \chi^{*} \gamma_{j} \mathcal{E} \mathcal{D}_{i} \mathcal{F}^{i j}+\frac{i}{4} \chi^{*} \gamma^{i j} \mathcal{E} \partial_{+} \mathcal{F}_{i j}+\frac{i}{2} \chi^{*} \mathcal{E} \mathcal{D}^{i} F_{i+} \\
& -\frac{i r}{4 \sqrt{2}} \chi^{*} \mathcal{E} \mathcal{G}^{i j} w_{i j}-\frac{i r}{2 \sqrt{2}} \chi^{*} \mathcal{E} \mathcal{F}^{i j} w_{i j} .
\end{aligned}
$$

The fourth term is identically zero because $\mathcal{G}_{i j}$ is antiself-dual.

Next, we obtain the supersymmetry variation

$$
\delta \psi=\frac{1}{12} \Gamma^{M N P} \varepsilon H_{M N P}
$$

in $4 \mathrm{~d}$. To this end, it is advantageous to first recast this in flat space indices,

$$
\delta \psi=\frac{1}{4} \Gamma^{\hat{i} \hat{j} \hat{j}} \varepsilon H_{\hat{i} \hat{j} \hat{+}}+\frac{1}{4} \Gamma^{\hat{i} \hat{j} \hat{j}} \varepsilon H_{\hat{i} \hat{j} \hat{\jmath}}+\frac{1}{2} \Gamma^{\hat{i} \hat{+} \hat{A}} \varepsilon H_{\hat{i} \hat{+} \hat{-} .} .
$$

Then it immediately follows that

$$
\delta \psi=\frac{1}{4} \tilde{\Gamma}^{i j} \Gamma^{\wedge} \varepsilon \mathcal{F}_{i j}+\frac{1}{2} \tilde{\Gamma}^{i} \Gamma^{\hat{+} \hat{}} \varepsilon F_{i+}
$$

which in terms of $4 \mathrm{~d}$ gamma matrices reads

$$
\delta \chi=\frac{1}{2 \sqrt{2}} \gamma^{i j} \mathcal{E} \mathcal{F}_{i j}-\frac{1}{2} \gamma^{i} \mathcal{E} F_{i+} .
$$

Then let us look at each term in turn in the fermionic action

$$
\begin{aligned}
\mathcal{L}_{F}= & \frac{i}{2} \chi^{*} \gamma^{i} \mathcal{D}_{i} \chi-\frac{i}{\sqrt{2}} \chi^{*} P_{-} \partial_{+} \chi \\
& +\frac{1}{r} \chi^{*} P_{+} \chi-\frac{i r}{16} \chi^{*} \gamma^{i j} P_{-} \chi w_{i j} .
\end{aligned}
$$

The variation of the first two derivative terms becomes, after using two types of Bianchi identities,

$$
\begin{aligned}
\delta\left(\mathcal{L}_{F}^{I}+\mathcal{L}_{F}^{I I}\right)= & \frac{i}{\sqrt{2}} \chi^{*} \gamma^{i} \mathcal{E} \mathcal{D}^{j} \mathcal{F}_{j i}-\frac{1}{2} \chi^{*} \mathcal{E} \mathcal{D}^{i} F_{i+} \\
& -\frac{i}{4} \chi^{*} \gamma^{i j} \mathcal{E} \partial_{+} \mathcal{F}_{i j}-\frac{1}{r 2 \sqrt{2}} \chi^{*} \gamma^{i j} \mathcal{E} \mathcal{F}_{i j} .
\end{aligned}
$$

The first line is exactly canceling corresponding terms in $\delta \mathcal{L}_{A}$. The variation of the two last mass terms gives

$$
\delta\left(\mathcal{L}_{F}^{I I}+\mathcal{L}_{F}^{I V}\right)=-\frac{1}{r} \chi^{*} \gamma^{i} \mathcal{E} F_{i+}-\frac{i r}{16 \sqrt{2}} \chi^{*} \gamma^{i j} \gamma^{k l} \mathcal{E} w_{i j} \mathcal{F}_{k l} .
$$

Ideally we had wanted these to cancel against the last term in $\delta \mathcal{L}_{A}$,

$$
\delta \mathcal{L}_{A}^{V I}=-\frac{i r}{2 \sqrt{2}} \chi^{*} \mathcal{E} w_{i j} \mathcal{F}^{i j}
$$

We do not seem to get a perfect cancellation, but let us note that we can rewrite the last term in $\delta\left(\mathcal{L}_{F}^{I I}+\mathcal{L}_{F}^{I V}\right)$ as

$$
\begin{aligned}
- & \frac{i r}{16 \sqrt{2}} \chi^{*}\left(\left\{\gamma^{i j}, \gamma^{k l}\right\}-\gamma^{k l} \gamma^{i j}\right) \mathcal{E} w_{i j} \mathcal{F}_{k l} \\
& =\frac{i r}{2 \sqrt{2}} \chi^{*} \mathcal{E} w_{i j} \mathcal{F}^{i j}+\frac{i r}{16 \sqrt{2}} \chi^{*} \gamma^{k l} \gamma^{i j} \mathcal{E} w_{i j} \mathcal{F}_{k l} \\
& =\frac{i r}{2 \sqrt{2}} \chi^{*} \mathcal{E} w_{i j} \mathcal{F}^{i j}+\frac{1}{r 2 \sqrt{2}} \chi^{*} \gamma^{i j} \mathcal{E} \mathcal{F}_{i j} .
\end{aligned}
$$

The first term cancels against $\delta \mathcal{L}_{A}^{V I}$ and the second term cancels the last term in $\delta\left(\mathcal{L}_{F}^{I}+\mathcal{L}_{F}^{I I}\right)$. The final result is that we have the following nonzero variation of the Lagrangian:

$$
\delta \mathcal{L}=-\frac{1}{r} \chi^{*} \gamma^{i} \mathcal{E} F_{i+} .
$$

Since the $6 \mathrm{~d}$ metric inverse $g^{i j}$ is equal to the $4 \mathrm{~d}$ metric inverse $H^{i j}$ and since the index $i$ in $F_{i+}=H_{i+-}$ can be extended to indices + and - without changing anything since $H_{++-}$and $H_{-+-}$are zero anyway, we can view $i$ as a $6 \mathrm{~d}$ index contracted by the $6 \mathrm{~d}$ metric. This means that we can write this result in terms of $6 \mathrm{~d}$ flat space indices as

$$
\delta \mathcal{L}=\frac{i}{r \sqrt{2}} \delta B_{\hat{+}}^{\hat{i}} H_{\hat{i} \hat{+} \hat{\imath}}
$$

and by using the self-duality relation

$$
H_{\hat{i} \hat{j} \hat{k}}=-\varepsilon_{\hat{i} \hat{j} \hat{k}}^{\hat{l}} F_{\hat{l} \hat{+} \hat{A}}
$$

we can further write this as

$$
\delta \mathcal{L}=\frac{i}{r \sqrt{2}} \varepsilon^{\hat{i} \hat{j} \hat{k} \hat{k} \hat{+} \hat{A}} \delta B_{\hat{i} \hat{+}} H_{\hat{j} \hat{k} \hat{l}} .
$$


Now we can change to $6 \mathrm{~d}$ curved space indices and then this becomes

$$
\delta \mathcal{L}=-\frac{i}{r \sqrt{2}} \varepsilon^{i j k l} H_{i j k} \delta B_{l+}
$$

where we define $\varepsilon^{i j k l}=-\varepsilon^{i j k l+-}$. We now wish to show that this can be expressed as a total variation of some topological term of the form

$$
\mathcal{L}_{\text {top }}=\varepsilon^{i j k l} H_{i j k} B_{l+}
$$

up to some constant factor. When we expand its variation, we find two types of terms,

$$
\delta \mathcal{L}_{\text {top }}=3 \varepsilon^{i j k l} \partial_{i} \delta B_{j k} B_{l+}+\varepsilon^{i j k l} H_{i j k} \delta B_{l+} .
$$

The first term here can be further be written as

$$
-3 \varepsilon^{i j k l} \delta B_{j k} \partial_{i} B_{l+}=-\frac{3}{2} \varepsilon^{i j k l} \delta B_{j k} H_{i l+}
$$

where we dropped a couple of total derivative terms. Now, if we change to flat space indices we see the emergence of an antiself-dual $H_{\hat{i} \hat{j} \hat{+}}=\mathcal{G}_{\hat{i} \hat{j}}$ and so what this term becomes is something that is proportional to $\delta B^{i j} \mathcal{G}_{i j}$ and this is zero, because $\delta B^{i j} \sim \chi^{*} \gamma^{i j} \mathcal{E}$ and we have that $\gamma^{i j} \mathcal{E G}_{i j}=0$ since $\mathcal{G}_{i j}$ is antiself-dual and $\mathcal{E}$ is Weyl. One way to see this is by noting that $\gamma^{i j} \mathcal{E} w_{i j}$ is nonzero where $w_{i j}$ is self-dual. This means that we are left with only the second term,

$$
\delta \mathcal{L}_{\text {top }}=\varepsilon^{i j k l} H_{i j k} \delta B_{l+}
$$

as we wanted to show. So by adding the topological term

$$
\mathcal{L}_{\text {top }}=\frac{i}{r \sqrt{2}} \varepsilon^{i j k l} H_{i j k} B_{l+}
$$

we find that its variation cancels the variation $\delta \mathcal{L}$ in (3.7) above.

Let us now study the matter part supersymmetry. The Lagrangian is

$$
\begin{aligned}
\mathcal{L}_{F}= & \frac{i}{2} \chi^{\dagger} \gamma^{i} D_{i} \chi-\frac{i}{\sqrt{2}} \chi^{\dagger} P_{-} \partial_{+} \chi+\frac{1}{r} \chi^{\dagger} P_{+} \chi \\
& -\frac{i}{2 r} \chi \gamma^{12} P_{-} \chi-\frac{i r}{2 \sqrt{2}} \kappa_{i} \chi^{\dagger} \gamma^{i} \partial_{+} \chi-\frac{1}{2} \kappa_{i} \chi^{\dagger} \gamma^{i} \chi .
\end{aligned}
$$

The supersymmetry variation is

$$
\delta \chi=-\gamma^{i} \tau^{A} \mathcal{E} \mathcal{D}_{i} \phi^{A}-\frac{2 i}{r} \tau^{A} \mathcal{E} \phi^{A}
$$

where we define

$$
\mathcal{D}_{i}=D_{i}-\kappa_{i} \partial_{y}, \quad \partial_{y}=\frac{r}{\sqrt{2}}\left(\partial_{+}-\partial_{-}\right) .
$$

Using this generalized derivative on the fermion, and the expansion where

$$
\partial_{-} \chi \rightarrow \frac{i \sqrt{2}}{r} \chi
$$

we find that the Lagrangian simplifies to

$$
\mathcal{L}_{F}=\frac{i}{2} \chi^{\dagger} \gamma^{i} \mathcal{D}_{i} \chi-\frac{i}{\sqrt{2}} \chi^{\dagger} P_{-} \partial_{+} \chi+\frac{1}{r} \chi^{\dagger} P_{+} \chi-\frac{i}{2 r} \chi \gamma^{12} P_{-} \chi .
$$

We thus need to carefully define the operator $\mathcal{D}_{i}$ acting on bosons and fermions respectively, as

$$
\begin{aligned}
& \mathcal{D}_{i} \phi=\partial_{i} \phi-\frac{r}{\sqrt{2}} \partial_{+} \phi, \\
& \mathcal{D}_{i} \chi=D_{i} \chi-\frac{r}{\sqrt{2}} \kappa_{i} \partial_{+} \chi+i \kappa_{i} \chi .
\end{aligned}
$$

Similarly then when this generalized derivative acts on the supersymmetry parameter, one then finds the following Killing spinor equation:

$$
\mathcal{D}_{i} \mathcal{E}=0
$$

We then get the supersymmetry variation

$$
\begin{aligned}
\delta \mathcal{L}_{F}= & -\frac{i}{2} \chi^{\dagger} \gamma^{i j} \tau^{A} \mathcal{E}\left[\mathcal{D}_{i}, \mathcal{D}_{j}\right] \phi^{A}-i \chi^{\dagger} \tau^{A} \mathcal{E} \mathcal{D}_{i}^{2} \phi^{A} \\
& -\frac{2 \sqrt{2}}{r} \chi^{\dagger} \tau^{A} \mathcal{E} \partial_{+} \phi^{A} \\
& +\frac{2 i}{r^{2}} \chi^{\dagger} \tau^{A} \mathcal{E} \phi^{A}-\frac{2}{r} \chi^{\dagger} \gamma^{12} \tau^{A} \mathcal{E} \phi^{A}
\end{aligned}
$$

Two terms cancel by using

$$
\left[\mathcal{D}_{i}, \mathcal{D}_{j}\right] \phi=-\frac{r}{\sqrt{2}} w_{i j} \partial_{+} \phi
$$

and

$$
\gamma^{12} \mathcal{E}=-i \mathcal{E}, \quad \gamma^{34} \mathcal{E}=-i \mathcal{E},
$$

and we get

$$
\delta \mathcal{L}_{F}=-i \chi^{\dagger} \tau^{A} \mathcal{E} \mathcal{D}_{i}^{2} \phi^{A}+\frac{4 i}{r^{2}} \chi^{\dagger} \tau^{A} \mathcal{E} \phi^{A} .
$$

Let us now turn to the scalar fields' Lagrangian

$$
\mathcal{L}_{S}=-\frac{1}{2}\left(\mathcal{D}_{i} \phi^{A}\right)^{2}-\frac{2}{r^{2}}\left(\phi^{A}\right)^{2} .
$$


Using the variation

$$
\delta \phi^{A}=i \chi^{\dagger} \tau^{A} \mathcal{E}
$$

we find that $\delta \mathcal{L}_{S}+\delta \mathcal{L}_{F}=0$.

Before turning to the non-Abelian case, let us first summarize the Abelian case. We have the Lagrangian

$$
\mathcal{L}=\mathcal{L}_{A}+\mathcal{L}_{\text {matter }}+\mathcal{L}_{\text {top }}
$$

where

$$
\begin{aligned}
\mathcal{L}_{A}= & \frac{1}{4}\left(\mathcal{F}^{i j} \mathcal{G}_{i j}+F^{i}{ }_{+} F_{i+}-\frac{r}{\sqrt{2}} \varepsilon^{i j k l} \mathcal{F}_{i j} F_{k+} \kappa_{l}\right), \\
\mathcal{L}_{\text {matter }}= & \frac{i}{2} \chi^{*} \gamma^{i} \mathcal{D}_{i} \chi-\frac{i}{\sqrt{2}} \chi^{*} P_{-} \partial_{+} \chi \\
& +\frac{1}{r} \chi^{*} P_{+} \chi-\frac{i r}{16} \chi^{*} \gamma^{i j} P_{-} \chi w_{i j} \\
& -\frac{1}{2}\left(\mathcal{D}_{i} \phi^{A}\right)^{2}-\frac{2}{r^{2}}\left(\phi^{A}\right)^{2}, \\
\mathcal{L}_{\text {top }}= & \frac{i}{r \sqrt{2}} \varepsilon^{i j k l} H_{i j k} B_{l+},
\end{aligned}
$$

and the supersymmetry variations

$$
\begin{aligned}
\delta \phi^{A} & =i \chi^{*} \tau^{A} \mathcal{E} \\
\delta \chi & =\frac{1}{2 \sqrt{2}} \gamma^{i j} \mathcal{E} \mathcal{F}_{i j}-\frac{1}{2} \gamma^{i} \mathcal{E} F_{i+}-\gamma^{i} \tau^{A} \mathcal{E} \mathcal{D}_{i} \phi^{A}-\frac{2 i}{r} \tau^{A} \mathcal{E} \phi^{A}, \\
\delta A_{i} & =-\frac{i r}{\sqrt{2}} \kappa_{i} \chi^{*} \mathcal{E}, \\
\delta A_{+} & =-i \chi^{*} \mathcal{E} \\
\delta F_{i+} & =-i \mathcal{D}_{i}\left(\chi^{*} \mathcal{E}\right), \\
\delta \mathcal{F}_{i j} & =-\frac{i r}{\sqrt{2}} \chi^{*} \mathcal{E} w_{i j}, \\
\delta \mathcal{G}_{i j} & =2 \sqrt{2} i \mathcal{D}_{i} \chi^{*} \gamma_{j} \mathcal{E}-\frac{i r}{\sqrt{2}} \chi^{*} \mathcal{E} w_{i j}-i \partial_{+}\left(\chi^{*} \gamma_{i j} \mathcal{E}\right) .
\end{aligned}
$$

We notice that the supersymmetry variation $\delta \mathcal{G}_{i j}$ is antiselfdual only on-shell, which naively means that imposing the antiself-dual constraint on $\mathcal{G}_{i j}$ breaks supersymmetry. On the other hand, we may also notice that the supersymmetry variation of $\delta \mathcal{F}_{i j}$ is self-dual off-shell. We can always decompose $\mathcal{F}_{i j}=\mathcal{F}_{i j}^{+}+\mathcal{F}_{i j}^{-}$into self-dual and antiselfdual parts as these transform in different representations under the $4 \mathrm{~d}$ rotation group and then we can impose the constraint that the antiself-dual part is zero, $\mathcal{F}_{i j}^{-}=0$ by hand as a constraint that we impose on top of the Lagrangian. At this stage, this constraint cannot be derived from the Lagrangian. This constraint does not break supersymmetry. From the coupling term $\sim \mathcal{F}_{i j} \mathcal{G}^{i j}$ in the Lagrangian we see that this constraint implies that only the antiself-dual part of $\mathcal{G}_{i j}$ enters in the Lagrangian, which is to say that $\mathcal{G}_{i j}$ is constrained to be antiself-dual and then we can change our viewpoint and take that constraint as a starting assumption and derive $\mathcal{F}_{i j}^{-}=0$ as an equation of motion.

To see whether a non-Abelian generalization is possible, let us start by replacing all derivatives with gauge covariant derivatives,

$$
\begin{aligned}
& \mathcal{D}_{i} \phi^{A}=D_{i} \phi^{A}-\kappa_{i} D_{y} \phi^{A}, \\
& D_{i} \phi^{A}=\partial_{i} \phi^{A}-i e\left[A_{i}, \phi^{A}\right], \\
& D_{y} \phi^{A}=\partial_{y} \phi^{A}-i e\left[A_{y}, \phi^{A}\right]
\end{aligned}
$$

in the supersymmetry variations. Then by noting that

$$
\left[\mathcal{D}_{i}, \mathcal{D}_{j}\right] \phi^{A}=-i e\left[\mathcal{F}_{i j}, \phi^{A}\right]
$$

we get

$$
\delta \mathcal{L}=-\frac{e}{2} \chi^{*} \gamma^{i j} \tau^{A} \mathcal{E}\left[\mathcal{F}_{i j}, \phi^{A}\right]
$$

To cancel this variation, one might be tempted to add the following term to the Lagrangian:

$$
\Delta \mathcal{L}=\frac{e}{\sqrt{2}} \chi^{*} \tau^{A}\left[\chi, \phi^{A}\right]
$$

But if we do that, then that term will upon a supersymmetry variation generate a host of new terms, such as

$$
\chi^{*} \gamma^{i} \mathcal{E}\left[\mathcal{D}_{i} \phi^{A}, \phi^{A}\right]
$$

but we cannot cancel this term by anything. The only candidate term $\left(\mathcal{D}_{i} \phi^{A}\right)^{2}$ does not work because the supersymmetry variation of the gauge potential $\mathcal{A}_{i}$ is vanishing, so it cannot give rise to something that is proportional to $\chi^{*} \gamma_{i} \mathcal{E}$. So we cannot cancel the variation (3.8) and therefore we shall not add any extra commutator terms to the Lagrangian.

Instead we shall modify the supersymmetry variation of $\mathcal{G}_{i j}$ by adding a term ${ }^{3}$

$$
\Delta \delta \mathcal{G}_{i j}=\frac{e}{2}\left[\chi^{*} \gamma_{i j} \tau^{A} \mathcal{E}, \phi^{A}\right]
$$

\footnotetext{
${ }^{3}$ This is in accordance with the Lambert-Papageorgakis theory [4], where

$$
\delta H_{M N P} \sim . .+\left[\phi^{A}, \bar{\psi}\right] \Gamma^{A} \Gamma_{M N P Q} \varepsilon v^{Q}
$$

if we notice that the only surviving combination of gamma matrices can be $\Gamma_{i j+-}$, which simply means that the commutator only enters in $H_{i j+}$, or in other words $\mathcal{G}_{i j}$.
} 
If we could make the gauge choice $A_{+}=0$ and then just forget about $\delta A_{+}$altogether, then since $\delta \mathcal{A}_{i}=0$, we would have no cubic term in the fermionic fields that could appear when we vary the gauge potential in the fermionic kinetic term. But imposing the gauge choice $A_{+}=0$ is unsatisfactory since this gauge choice breaks supersymmetry by itself. We can avoid this problem of gauge fixing by reducing supersymmetry by another half. We then impose the Weyl projection

$$
\tau^{5} \mathcal{E}=\mathcal{E}
$$

Then we have the supersymmetry variation

$$
\delta \phi^{5}=i \chi^{*} \mathcal{E}
$$

and we see that the combination $A_{+}-\phi^{5}$ is a supersymmetric invariant,

$$
\delta\left(A_{+}-\phi^{5}\right)=0 .
$$

We then obtain a supersymmetric Lagrangian by simply adding commutator terms that involve $\phi^{5}$ for each place where there is a gauge field $A_{+}$. Such commutator terms are of course gauge invariant by themselves. But we can repackage these terms into a new derivative

$$
\mathcal{D}_{+}=D_{+}+i e\left[\phi^{5}, \bullet\right]
$$

where $D_{+}=\partial_{+}-i e\left[A_{+}, \bullet\right]$. One may worry that an ordinary derivative acts on a fermionic field, but that is just because of how we have set up our Lagrangian. We have already taken into account all those curvature corrections when we analyzed the Abelian case and those curvature corrections will not be affected in any significant way by the non-Abelian generalization. We now obtain a full supersymmetric non-Abelian Lagrangian by replacing every occurrence of $\partial_{+}$with $\mathcal{D}_{+}$as we defined it above (with an ordinary derivative $\partial_{+}$rather than a curvature covariant $\nabla_{+}$). There is now at this stage no need to impose any gauge fixing condition on $A_{+}$.

The $B \wedge H$ term is straightforwardly generalized to the non-Abelian case as $B^{a} \wedge H^{a}$ where $a$ is the adjoint gauge group index. The supersymmetry variation of $\tilde{B}_{i+}$ is similarly generalized by just attaching that adjoint gauge group index as $\delta B_{i+}^{a}=-i \sqrt{2}\left(\chi^{a}\right)^{*} \gamma_{i} \mathcal{E}$. We also assume the duality relation is generalized to the non-Abelian case as $H_{i j k}^{a}=\varepsilon_{i j k l} F_{l+}^{a}$.

As we did not put any component of the fermionic field to zero here, as we did for the case of time reduction, we do not expect our $5 \mathrm{~d}$ Lagrangian will be possible to derive by turing on an R-gauge field in some dual formulation. In particular, we do not expect the closure relations will be of a standard form.
It would be interesting to see if our null reduction here can be generalized to the generic null geometries of [3]. If that is the case, then that would mean that we would have a supersymmetric five-dimensional Lagrangian description for M5 branes on any Lorentzian geometry that has a Killing vector along a null direction.

\section{DISCUSSION}

For any supersymmetric Abelian theory on a circle bundle, we have shown by a general argument that we can always obtain a corresponding supersymmetric Lagrangian in one dimension lower by keeping nonzero KK fermionic modes. We have demonstrated this explicitly for the Abelian M5 brane on $\mathbb{R} \times S^{5}$ by first reducing along the time direction and next along a null direction.

The main problem we would like to focus on however is not the Abelian case, which is somewhat trivial, but the nonAbelian generalization. A non-Abelian generalization of the M5 brane Lagrangian may not exist. We have also found that it is very difficult to find a non-Abelian generalization of the dimensionally reduced theory when we keep a nonzero fermionic KK mode. But this problem is due to the fact that our reduction is in general not consistent with supersymmetry. So even if there had been a non-Abelian Lagrangian in 6d we would still have troubles in general to find a corresponding supersymmetric Lagrangian in $5 \mathrm{~d}$ in this way.

Nevertheless, we have seen that a non-Abelian Lagrangian could be found by reducing the amount of supersymmetry sufficiently much for the two cases of time and null reductions on $\mathbb{R} \times S^{5}$.

It would be interesting if our result can be generalized to arbitrary null geometries since then we would have a large class of supersymmetric $5 \mathrm{~d}$ Lagrangians that might be helpful to get a better understanding of the non-Abelian M5 brane theory as we then can make use of this supersymmetry to quantize these $5 \mathrm{~d}$ theories. It would also be nice if one could recover the Lagrangian in [8] as a special case from such a more general supersymmetric Lagrangian.

In our dimensional reduction we keep nonzero KK modes for the fermionic fields and zero modes for the bosonic fields. One may want to gain some intuition for what this means by putting M5 branes on $\mathbb{R}^{1,4} \times S^{1}$ and reduce down to $\mathbb{R}^{1,4}$. Then to relate to our work, one may impose an antiperiodic boundary condition for the fermions around the $S^{1}$. If we assume this, then we have only the odd KK modes for the antiperiodic fermions and only the even KK modes for the periodic bosons. By looking at the theory at energies below the second $\mathrm{KK}$ mode, dimensional reduction could amount to keeping bosonic zero modes and the first excited nonzero fermionic KK modes, although this dimensional reduction would not be as good as usual dimensional reduction since we would never get very far from the second KK mode if we consider excitations above the first KK mode, so the validity of such a dimensional reduction may be subject to some 
criticism. Nevertheless, such an example may provide some intuition for the dimensional reduction that we have studied in this paper. However, this is not really the kind of situation that we have studied in this paper, and the intuition this flat spacetime example may provide us is quite misleading. If we impose antiperiodic boundary conditions around $S^{1}$ then all supersymmetry is broken, since in flat space, the supersymmetry parameter is constant along $S^{1}$. The supersymmetry preserving boundary condition will be the periodic boundary condition and then we should keep the fermionic zero mode and study the usual dimensional reduction.

The upshot of this example is that we cannot use much of our flat spacetime intuition to understand the dimensional reduction that we have studied in this paper. We need to put the M5 brane on a curved spacetime that removes the zero mode around $S^{1}$ in the supersymmetry parameter. In a curved spacetime there is no clear cut relation between energy levels and KK mode numbers when we compare different kinds of fields. One example that we considered was dimensional reduction on $\mathbb{R} \times S^{5}$ down to $S^{5}$. Unfortunately this is a subtle example from a physics viewpoint, since dimensional reduction is along time and moreover, time is naturally noncompact. Nevertheless, the supersymmetry parameter has an oscillatory behavior along time in Lorentzian signature in this example and we can use this fact to obtain a 5d SYM theory on $S^{5}$. Although we kept a nonzero KK mode for the fermionic field, we ended up with the same $5 \mathrm{~d}$ Lagrangian as we would get if instead we had started with a $6 \mathrm{~d}$ theory with a background R-gauge field turned on along the time direction chosen so that the supersymmetry parameter has a zero mode and we perform usual dimensional reduction. This example indicates that in curved spacetime there may not be a simple correspondence between the KK level and the energy level as there is in flat spacetime. The energy level for different fields may involve different kinds of curvature corrections and supergravity background fields, and it may be that a nonzero fermionic mode has the exact same energy as a bosonic zero mode so that these different modes can be related by supersymmetry.

Inspired by a conjectured relation between $5 \mathrm{~d}$ maximally SYM on $\mathbb{R}^{1,4}$ and M5 branes on $\mathbb{R}^{1,4} \times S^{1}[9,10]$, one may ask whether some 5d YM theory could also capture all of a corresponding M5 brane theory on a more general $U(1)$ bundle geometry. But to generalize the conjecture in $[9,10]$ to $U(1)$ bundles, one needs a further specification in curved spacetimes because we may have a supersymmetric M5 brane for either a periodic or for an antiperiodic spin structure around the $U(1)$ isometry circle. We will thus have to distinguish between these two different boundary conditions or spin structures on the $U(1)$. When the spin structure is even, the supersymmetric boundary condition is the periodic boundary condition and if the supersymmetry parameter is constant along the
$U(1)$ direction, then we can perform usual dimensional reduction by keeping only the zero modes of bosonic and fermionic fields and arrive at the SYM theory that was discussed in, for instance, [11]. When the spin structure is odd, the supersymmetric boundary condition is antiperiodic and the usual dimensional reduction removes the fermionic modes. We get a purely bosonic YM theory in $5 \mathrm{~d}$. However, in this paper we have shown that it is possible in some cases to get a supersymmetric $5 \mathrm{~d}$ YM if instead we keep the first nonzero fermionic modes. Which of these two 5d theories (if any) would correspond to the non-Abelian M5 brane for the case of an antiperiodic spin structure? It seems difficult to generate the missing fermionic KK modes from a purely bosonic YM theory by quantizing the fluctuation fields around its instanton particles. In a supersymmetric YM the fermionic KK modes arise as fermionic zero modes when one quantizes the fluctuations around an instanton particle. One might speculate that perhaps the supersymmetric $5 \mathrm{~d}$ SYM theory that we have constructed by performing a null reduction could capture all of the non-Abelian M5 brane theory with an odd spin structure, but this remains to be seen.

We have motivated our route to get from the Abelian M5 brane to the Abelian 5d SYM that has a unique nonAbelian generalizations that under certain circumstances is also supersymmetric. The next question is of course whether we can use this non-Abelian $5 \mathrm{~d}$ supersymmetric YM theory to get information about the corresponding non-Abelian M5 brane theory. We should not claim that the $5 \mathrm{~d}$ theory is the non-Abelian M5 brane. First, this depends on whether the conjecture in $[9,10]$ is correct, and second whether that conjecture can be generalized to other geometries such as those that we have studied in this paper. Still one may ask a more modest question, whether one can quantize the $5 \mathrm{~d}$ theory that we have obtained. A similar question has been studied in $[2,12]$ (and in many papers following these two seminal works) where one studied $5 \mathrm{~d}$ SYM on $S^{5}$ and computed its partition function by using localization. A match with corresponding dual supergravity computation was found, which suggested that this location method seemed to give the M5 brane partition function. For our null reduction, since we have a supersymmetric $5 \mathrm{~d}$ theory, perhaps we can apply the localization technique also on this $5 \mathrm{~d}$ supersymmetric theory. However, we need to impose at least one self-duality constraint by hand, on either $\mathcal{F}_{i j}$ or $\mathcal{G}_{i j}$. The question is whether it is possible to handle this constraint in a way that is consistent with such a localization computation. Another way to approach the quantum theory is by using the method of discrete light-cone quantization. That amounts to deriving a corresponding quantum mechanics Lagrangian on the instanton moduli space that one may subsequently try to quantize [13]. However this approach will be difficult to carry out if the instanton moduli space is too complicated. 


\section{ACKNOWLEDGMENTS}

I would like to thank Dongsu Bak for discussions. This work was supported in part by NRF Grants No. 2020R1A2B5B01001473 and No. 2020R1I1A1A01052462.

\section{APPENDIX A: A 6D FORMULATION OF 5D SYM}

There is a $6 \mathrm{~d}$ formulation of $5 \mathrm{~d}$ SYM where one introduces a vector field $v^{M}$ and requires all fields to have vanishing Lie derivatives along that vector field [4-6]. We did not make explicit use of this $6 \mathrm{~d}$ formulation of 5d SYM. But it was this formulation that originally motivated us to search for consistent supersymmetric truncations, and the two cases that we have studied in this paper can be at least intuitively quite clearly understood by looking at this formulation of the theory where they emerge as the Weyl projections (A8) and (A9) respectively.

The 6d supersymmetry variations look like a nonAbelian generalization of the Abelian M5 brane, but of course there is a catch. Namely we do not have closure relations satisfied for these variations, unless two terms vanish, namely the terms in (A3) and (A4). Let us present this in detail. The supersymmetry variations are given by

$$
\begin{aligned}
\delta \phi^{A}= & i \bar{\varepsilon} \Gamma^{A} \psi, \\
\delta H_{M N P}= & 3 i D_{P}\left(\bar{\varepsilon} \Gamma_{M N} \psi\right)+e \bar{\varepsilon} \Gamma_{M N P Q} \Gamma^{A}\left[\psi, \phi^{A}\right] v^{Q}, \\
\delta A_{N}= & i \bar{\varepsilon} \Gamma_{N P} \psi v^{P}, \\
\delta \psi= & \frac{1}{12} \Gamma^{M N P} \varepsilon H_{M N P}+\Gamma^{M} \Gamma^{A} \varepsilon D_{M} \phi^{A}-4 \Gamma^{A} \eta \phi^{A} \\
& -\frac{i e}{2} \Gamma_{M} \Gamma^{A B} \varepsilon\left[\phi^{A}, \phi^{B}\right] v^{M} .
\end{aligned}
$$

Here

$$
D_{M} \phi^{A}=\partial_{M} \phi^{A}-i e\left[A_{M}, \phi^{A}\right]+V_{M}^{A B} \phi^{B}
$$

where $V_{M}$ is an R-gauge field, and

$$
\begin{aligned}
& D_{M} \varepsilon=\Gamma_{M} \eta-\frac{1}{8} \Gamma^{A} \Gamma^{R S T} \Gamma_{M} \varepsilon T_{R S T}^{A}, \\
& D_{M} \bar{\varepsilon}=-\bar{\eta} \Gamma_{M}-\frac{1}{8} \bar{\varepsilon} \Gamma_{M} \Gamma^{R S T} \Gamma^{A} T_{R S T}^{A} .
\end{aligned}
$$

Here $v^{M}$ is a Killing vector field and $\mathcal{L}_{v}$ denotes the Lie derivative along this Killing vector field. We will impose the gauge condition

$$
A_{M} v^{M}=0
$$

which is a very natural gauge condition if we think on $A_{M}$ as $B_{M N} v^{N}$. Now this correspondence is at present unknown to us for the non-Abelian case where $H_{M N P}$ is all that we have.
We would like to know how to express the theory in terms of some non-Abelian gauge potential $B_{M N}$ but at present we do not have such a formulation. Nevertheless, the gauge potential will be assumed to satisfy the gauge condition (A2).

We define the $6 \mathrm{~d}$ chirality matrix

$$
\Gamma=\Gamma^{\hat{0} \hat{1} \hat{2} \hat{3} \hat{5} \hat{5}}
$$

in flat tangent space and we assume that the spinor and supersymmetry parameter have opposite chiralities

$$
\Gamma \psi=\psi, \quad \Gamma \varepsilon=-\varepsilon .
$$

We use $11 \mathrm{~d}$ gamma matrices where $\Gamma_{M}$ denote spacetime gamma matrices for $M=0,1, \ldots, 5$ and $\Gamma_{A}$ denote five transverse space gamma matrices for $A=1,2,3,4,5$ and these anticommute, $\left\{\Gamma_{M}, \Gamma_{A}\right\}=0$.

For the closure computation of these supersymmetry variations, we define

$$
S^{M}=\bar{\varepsilon} \Gamma^{M} \varepsilon
$$

and the gauge parameter

$$
\Lambda=-i \bar{\varepsilon} \Gamma_{Q} \Gamma^{A} \varepsilon \phi^{A} v^{Q}
$$

and we assume that $\varepsilon$ is a commuting spinor, since that simplifies the closure computation a bit yet without imposing any restrictions.

The superconformal algebra in curved space is

$$
\delta_{\varepsilon}^{2}=-i \mathcal{L}_{S}-2 i \mathcal{W}-2 \bar{\varepsilon} \Gamma^{A B} \eta R^{A B}+\delta_{\text {gauge }}
$$

where

$$
R^{A B}=\frac{i}{2} \Gamma^{A B}, \quad R_{C D}^{A B}=2 i \delta_{C D}^{A B},
$$

and

$$
\mathcal{W}_{\phi^{A}}=2, \quad \mathcal{W}_{\psi}=\frac{5}{2}, \quad \mathcal{W}_{H_{M N P}}=0
$$

are the Weyl weights.

As always with closure relations, we can express these in terms of conventional Lie derivatives $\mathcal{L}_{S}$, or in terms of gauge covariant Lie derivatives $\mathbb{L}_{S}$. These are related as

$$
\begin{aligned}
-i \mathbb{L}_{S} \phi^{A} & =-i \mathcal{L}_{S} \phi^{A}-i e\left[\phi^{A}, \Delta \lambda\right]-i S^{M} V_{M}^{A B} \phi^{B}, \\
-i \mathbb{\complement}_{S} \psi & =-i \mathcal{L}_{S} \psi-i e[\psi, \Delta \lambda]-\frac{i}{4} S^{M} V_{M}^{A B} \Gamma^{A B} \psi, \\
-i \mathbb{\complement}_{S} H_{M N P} & =-i \mathcal{L}_{S} H_{M N P}-i e\left[H_{M N P}, \Delta \lambda\right], \\
-i \mathbb{\complement}_{S} A_{M} & =-i S^{N} F_{N M} \\
& =-i \mathcal{L}_{S} A_{M}+D_{M}(\Delta \lambda),
\end{aligned}
$$


where $\Delta \lambda=i S^{M} A_{M}$. We thus see that from $\mathbb{R}_{S}$ we get $\mathcal{L}_{S}$ plus some extra gauge transformation and R-rotation.

If we assume that $\varepsilon$ is commuting, then we find the following closure relations:

$$
\begin{aligned}
\delta^{2} \phi^{A}= & -i \mathbb{\complement}_{S} \phi^{A}-4 i \bar{\varepsilon} \eta \phi^{A}-4 i \bar{\varepsilon} \Gamma^{A B} \eta \phi^{B}-i e\left[\phi^{A}, \Lambda\right], \\
\delta^{2} H_{M N P}= & -i \mathbb{L}_{S} H_{M N P}+3 i D_{M}\left(S^{T}\left(H_{N P T}^{-}-6 \phi^{A} T_{N P T}^{A}\right)\right) \\
& -4 i S^{T} D_{[P} H_{M N T]}-e \varepsilon_{M N P Q R S} \bar{\varepsilon} \Gamma^{S} \varepsilon\left[D_{R} \phi^{A}, \phi^{A}\right] v^{Q}-\frac{i e}{2} \bar{\varepsilon} \Gamma^{V} \varepsilon \varepsilon_{M N P Q U V} \bar{\psi}^{b} \Gamma^{U} \psi^{a} v^{Q} \\
& +3 e \bar{\varepsilon} \Gamma_{P} \Gamma^{A} \varepsilon\left[H_{M N Q} v^{Q}-F_{M N}, \phi^{A}\right]-i e\left[H_{M N P}, \Lambda\right]+\frac{e}{2} \mathcal{L}_{v}\left(\bar{\varepsilon} \Gamma^{A B} \Gamma_{M N P} \varepsilon\right)\left[\phi^{A}, \phi^{B}\right], \\
\delta^{2} \psi= & -i \mathbb{L}_{S} \psi-2 i \bar{\eta} \varepsilon \frac{5}{2} \psi-i \bar{\eta} \Gamma^{A B} \varepsilon \Gamma^{A B} \psi-i e[\psi, \Lambda] \\
& +\frac{3 i}{8} S^{Q} \Gamma_{Q}\left(\Gamma^{P} D_{P} \psi+\frac{1}{4} \Gamma^{R S T} \Gamma^{A} \psi T_{R S T}^{A}-i e \Gamma_{M} \Gamma^{A}\left[\psi, \phi^{A}\right] v^{M}\right) \\
& +2 i c^{Q B} \Gamma_{Q} \Gamma^{B}\left(\Gamma^{P} D_{P} \psi+\frac{1}{4} \Gamma^{R S T} \Gamma^{A} \psi T_{R S T}^{A}-i e \Gamma_{M} \Gamma^{A}\left[\psi, \phi^{A}\right] v^{M}\right), \\
\delta^{2} A_{M}= & -i \mathbb{L}_{S} A_{M}+D_{M}\left(-i \bar{\varepsilon} \Gamma_{N} \Gamma^{A} \varepsilon \phi^{A} v^{N}\right)+i S^{T} F_{T M}-i S^{T}\left(H_{M N T}^{+}+6 T_{M N P}^{A} \phi^{A}\right) v^{N}+i \mathcal{L}_{v}\left(\bar{\varepsilon} \Gamma_{M} \Gamma^{A} \varepsilon \phi^{A}\right) .
\end{aligned}
$$

Apart from the term

$$
\frac{e}{2} \mathcal{L}_{v}\left(\bar{\varepsilon} \Gamma^{A B} \Gamma_{M N P} \varepsilon\right)\left[\phi^{A}, \phi^{B}\right]
$$

in $\delta^{2} H_{M N P}$ and the term

$$
i \mathcal{L}_{v}\left(\bar{\varepsilon} \Gamma_{M} \Gamma^{A} \varepsilon \phi^{A}\right)
$$

in $\delta^{2} A_{M}$, we can now obtain closure up to a gauge transformation with gauge parameter $\lambda=-i \bar{\varepsilon} \Gamma_{M} \Gamma^{A} \varepsilon \phi^{A} v^{M}$ if certain equations of motion are satisfied. Closure on $H_{M N P}$ requires the following equations of motion:

$$
\begin{gathered}
H_{N P T}^{-}-6 \phi^{A} T_{N P T}^{A}=0, \\
H_{M N Q} v^{Q}-F_{M N}=0,
\end{gathered}
$$

and closure on $A_{M}$ requires the equation of motion

$$
F_{T M}-\left(H_{M N T}^{+}+6 T_{M N P}^{A}\right) v^{N}=0 .
$$

By adding $0=H_{N P T}^{-}-6 \phi^{A} T_{N P T}^{A}$ to $H_{M N T}^{+}+6 T_{M N P}^{A} \phi^{A}$ we get $H_{M N T}=H_{M N T}^{+}+H_{M N T}^{-}$and (A7) reduces to (A6). Of course the presence of the terms (A3) and (A4) means that these $6 \mathrm{~d}$ supersymmetry variations do not close, unless both these terms vanish. One way to make these two terms vanish is by requiring the Lie derivative vanishes on every field and also on the supersymmetry parameter, $\mathcal{L}_{v} \varepsilon=0$, where $\mathcal{L}_{v}$ denotes the Lie derivative along $v^{M}$. This is the usual dimensional reduction along the vector field $v^{M}$.

Could there be some other ways to achieve closure? At least for the first term (A3), we can make that term disappear without requiring $\mathcal{L}_{v} \varepsilon=0$. To see this more clearly, let us notice that a corresponding commutator term sits in the supersymmetry variation of the $(2,0)$ tensor multiplet fermion $\psi$ as

$$
\delta \psi=\ldots-\frac{i e}{2} \Gamma_{M} \Gamma^{A B} \varepsilon\left[\phi^{A}, \phi^{B}\right] v^{M}
$$

and here we can see two ways for this commutator term to vanish.

One is by just keeping one scalar field, say $\phi^{5}$, and reduce supersymmetry by imposing the R-symmetry Weyl projection

$$
\Gamma^{A=5} \varepsilon=\varepsilon
$$

and discarding the hypermultiplet. Of course, with just one scalar field, there will be no nontrivial commutator term $\left[\phi^{A}, \phi^{B}\right]$, but having to discard the hypermuliplet is of course unsatisfactory.

The other way to get rid of this term is by taking $v^{M}$ to be a null vector and imposing the Weyl projection

$$
\Gamma_{M} \varepsilon v^{M}=0
$$

and again this commutator term will vanish. The advantage of the null reduction is clearly that we can keep the full tensor multiplet structure with the five scalar fields intact.

\section{APPENDIX B: THE EUCLIDEAN M5 BRANE}

So far we have discussed only the Lorentzian M5 brane. But if we eventually would like to study the M5 brane on say $S^{6}$, then we will need to understand what the Euclidean M5 brane really means in terms of its tensor multiplet structure and its supersymmetry. So here we will clarify this point. First we begin with what is familiar to us though, namely the Lorentzian tensor multiplet, and then we seek a 
way to modify this so that we can allow a Euclidean signature.

\section{The Lorentzian $(2,0)$ and $(0,2)$ tensor multiplets}

We begin with Lorentzian $S O(1,5) \times S O(5) \subset S O(1,10)$ where we have the Dirac conjugate $\bar{\varepsilon}=\varepsilon^{\dagger} \Gamma^{0}$ and the Majorana condition $\bar{\varepsilon}=\varepsilon^{T} C$ that in terms of Weyl components reads $\varepsilon^{\mp \dagger} \Gamma=\varepsilon^{\mp T} C$ and hence is compatible with Weyl projection $\varepsilon^{+}=0$. We then have the chiral $(2,0)$ tensor mutliplet

$$
\begin{aligned}
\delta \phi^{+A} & =i \bar{\varepsilon} \Gamma^{A} \psi^{+} \\
\delta B_{M N}^{+} & =i \bar{\varepsilon} \Gamma_{M N} \psi^{+} \\
\delta \psi^{+} & =\frac{1}{12} \Gamma^{M N P} \varepsilon H_{M N P}^{+}+\Gamma^{M} \Gamma^{A} \varepsilon D_{M} \phi^{+A}-4 \Gamma^{A} \eta \phi^{+A} .
\end{aligned}
$$

We may also consider the antichiral $(0,2)$ tensor multiplet

$$
\begin{aligned}
\delta \phi^{-A} & =i \bar{\varepsilon} \Gamma^{A} \psi^{-}, \\
\delta B_{M N}^{-} & =i \bar{\varepsilon} \Gamma_{M N} \psi^{-}, \\
\delta \psi^{-} & =\frac{1}{12} \Gamma^{M N P} \varepsilon H_{M N P}^{-}+\Gamma^{M} \Gamma^{A} \varepsilon D_{M} \phi^{-A}-4 \Gamma^{A} \eta \phi^{-A},
\end{aligned}
$$

and if we put them together we can write a Lagrangian,

$$
\begin{aligned}
\mathcal{L}_{(2,0)+(0,2)}= & -\frac{1}{24} H_{M N P}^{2}+\mathcal{L}^{+}+\mathcal{L}^{-}, \\
\mathcal{L}^{ \pm}= & -\frac{1}{2}\left(D_{M} \phi^{ \pm A}\right)^{2}-\frac{1}{2} \mu^{A B} \phi^{ \pm A} \phi^{ \pm B} \\
& +\frac{i}{2} \bar{\psi}^{ \pm} \Gamma^{M} D_{M} \psi^{ \pm}+\frac{i}{8} \bar{\psi}^{ \pm} \Gamma^{M N P} \Gamma^{A} \psi^{ \pm} T_{M N P}^{\mp A},
\end{aligned}
$$

that is invariant under both the $(2,0)$ and the $(0,2)$ superconformal symmetries where the corresponding supersymmetry parameters satisfy

$$
D_{M} \varepsilon^{\mp}=\Gamma_{M} \eta^{ \pm}-\frac{1}{8} \Gamma^{A} \Gamma^{R S T} \Gamma_{M} \varepsilon^{\mp} T_{R S T}^{\mp A} .
$$

These Killing spinor equations are compatible with the Majorana conditions $\varepsilon^{\mp \dagger} \Gamma^{0}=\varepsilon^{\mp T} C$ only if we require that

$$
\left(\eta^{ \pm}\right)^{\dagger} \Gamma^{0}=\left(\eta^{ \pm}\right)^{T} C, \quad\left(T_{R S T}^{\mp A}\right)^{*}=T_{R S T}^{\mp A} .
$$

To see that we use $\left(\Gamma^{M}\right)^{\dagger}=\Gamma^{0} \Gamma^{M} \Gamma^{0}$.

\section{The Euclidean $(2,2)$ tensor multiplet}

We change to Euclidean signature $S O(6) \times S O(5) \subset$ $S O(6,5)$ by defining the Dirac conjugate as $\bar{\varepsilon}=\varepsilon^{\dagger} \Gamma$. We impose the $11 \mathrm{~d}$ Majorana condition $\bar{\varepsilon}=\varepsilon^{T} C$ with that new Dirac conjugate. In terms of Weyl components, this reads $\left(\varepsilon^{ \pm}\right)^{\dagger} \Gamma=\left(\varepsilon^{\mp}\right)^{T} C$ and we cannot impose the 6d Weyl condition. We have the Euclidean nonchiral $(2,2)$ multiplet

$$
\begin{aligned}
\delta \phi^{ \pm A} & =i \varepsilon^{ \pm \dagger} \Gamma \Gamma^{A} \psi^{ \pm} \\
\delta B_{M N} & =\varepsilon^{\dagger} \Gamma \Gamma_{M N} \psi \\
\delta \psi^{ \pm} & =\frac{i}{12} \Gamma^{M N P} \varepsilon^{\mp} H_{M N P}+\Gamma^{M} \Gamma^{A} \varepsilon^{\mp} D_{M} \phi^{ \pm A}-4 \Gamma^{A} \eta^{ \pm} \phi^{ \pm A}
\end{aligned}
$$

where we have removed a factor of $i$ from the variation $\delta B_{M N}^{ \pm}$to make the variation Hermitian by using the Majorana condition. We also multiplied $H_{M N P}$ by a factor of $i$ in $\delta \psi$ to make the variation compatible with the Majorana condition with $H_{M N P}$ real. Because of this $i$, there is a change of sign in the kinetic term for the tensor field and the Lagrangian is

$$
\mathcal{L}_{(2,2)}=\frac{1}{24} H_{M N P}^{2}+\mathcal{L}^{+}+\mathcal{L}^{-}
$$

where the matter part looks identical with that of the Lorentzian $(2,0)+(0,2)$ theory if we write the Dirac conjugates as $\psi^{T} C$. But if we use the new Majorana condition then it will look like

$$
\begin{aligned}
\mathcal{L}^{ \pm}= & -\frac{1}{2}\left(D_{M} \phi^{ \pm A}\right)^{2}-\frac{1}{2} \mu^{A B} \phi^{ \pm A} \phi^{ \pm B} \\
& +\frac{i}{2} \psi^{\mp \dagger} \Gamma \Gamma^{M} D_{M} \psi^{ \pm}-\frac{1}{8} \psi^{\mp \dagger} \Gamma \Gamma^{M N P} \Gamma^{A} \psi^{ \pm} T_{M N P}^{\mp A}
\end{aligned}
$$

where we also multiplied $T_{M N P}^{A}$ with a factor of $i$, which is in line with having the same factor of $i$ multiplying $H_{M N P}$. We may notice that the chiral parts $H_{M N P}^{ \pm}$will be complex fields, but the sum, $H_{M N P}=H_{M N P}^{+}+H_{M N P}^{-}$will be real. This observation may be used for holomorphic factorization of the partition function in Euclidean signature. We get back to the $(2,0)$ tensor multiplet by replacing $\psi^{-\dagger} \Gamma$ with $\psi^{+T} C$. Once we have done that replacement, we drop the 11d Majorana condition and impose the Weyl projection $\psi^{-}=0$. Then $\mathcal{L}^{+}$will become identical with $\mathcal{L}_{(2,0)}$ [although we are now in signature $S O(6,5)]$. We can do the corresponding replacements for the $(0,2)$ theory. These two supersymmetries do not mix once we formulate the theory in terms of $\psi^{T} C$. The supersymmetry parameters satisfy

$$
D_{M} \varepsilon^{\mp}=\Gamma_{M} \eta^{ \pm}-\frac{i}{8} \Gamma^{A} \Gamma^{R S T} \Gamma_{M} \varepsilon^{\mp} T_{R S T}^{ \pm A}
$$

where consistency with the Majorana condition implies that

$$
\eta^{ \pm \dagger} \Gamma=-\eta^{ \pm T} C, \quad\left(T_{M N P}^{ \pm A}\right)^{\dagger}=T_{M N P}^{ \pm A}
$$




\section{APPENDIX C: THE MAJORANA CONDITION IN VARIOUS DIMENSIONS}

The 11d Majorana condition is

$$
\bar{\psi}=\psi^{T} C
$$

where we define $\bar{\psi}=\psi^{\dagger} \Gamma^{t}$. We will represent the $11 \mathrm{~d}$ gamma matrices as

$$
\begin{aligned}
\Gamma^{t} & =i\left(\sigma^{2}\right)^{A}{ }_{B} \delta_{\beta}^{\alpha} \delta_{\dot{\beta}}^{\dot{\alpha}}, \\
\Gamma^{m} & =\left(\sigma^{1}\right)^{A}{ }_{B}\left(\gamma^{m}\right)^{\alpha}{ }_{\beta} \delta_{\dot{\beta}}^{\dot{\alpha}}, \\
\Gamma^{A} & =\left(\sigma^{3}\right)^{A}{ }_{B} \delta_{\beta}^{\alpha}\left(\tau^{A}\right)^{\dot{\alpha}}{ }_{\dot{\beta}} .
\end{aligned}
$$

The charge conjugation matrix is

$$
C=\varepsilon_{A B} C_{\alpha \beta} C_{\dot{\alpha} \dot{\beta}}
$$

Hence the 11d Majorana condition is

$$
\left(\psi^{A \alpha \dot{\alpha}}\right)^{*} i\left(\sigma^{2}\right)^{A}{ }_{B}=\psi^{B \dot{\beta} \dot{\beta}} \varepsilon_{B A} C_{\beta \alpha} C_{\dot{\beta} \dot{\alpha}} .
$$

The $6 \mathrm{~d}$ chirality matrix is

$$
\Gamma=\left(\sigma^{3}\right)^{A}{ }_{B} \delta_{\beta}^{\alpha} \delta_{\dot{\beta}}^{\dot{\alpha}} .
$$

So if we define $\varepsilon_{+-}=1$, then we find

$$
\left(\psi^{+\alpha \dot{\alpha}}\right)^{*}=C_{\alpha \beta} C_{\dot{\alpha} \dot{\beta}} \psi^{+\beta \dot{\beta}}, \quad\left(\varepsilon^{-\alpha \dot{\alpha}}\right)^{*}=C_{\alpha \beta} C_{\dot{\alpha} \dot{\beta}} \varepsilon^{-\beta \dot{\beta}} .
$$

If we reduce to $5 \mathrm{~d}$ then we have the spinor zero modes that satisfy the above Majorana condition, but the chirality has lost its significance so we choose to not display it when we work in $5 \mathrm{~d}$ language, so instead of writing $\psi^{+\alpha \dot{\alpha}}$, we will just write $\psi^{\alpha \dot{\alpha}}$ when this is a $5 \mathrm{~d}$ spinor.

From

$$
D_{M} \varepsilon=\Gamma_{M} \eta
$$

we get

$$
D_{M} \varepsilon^{\dagger} \Gamma^{t}=-\eta^{\dagger} \Gamma^{t} \Gamma_{M}, \quad D_{M} \varepsilon^{T} C=-\eta^{T} C \Gamma_{M} .
$$

Applying the Majorana condition on the left-hand side of the first equation, we get

$$
D_{M} \varepsilon^{T} C=-\eta^{\dagger} \Gamma^{t} \Gamma_{M}
$$

and by identifying this with the right-hand side of the second equation, we conclude that

$$
\eta^{\dagger} \Gamma^{t}=\eta^{T} C
$$

\section{APPENDIX D: METRIC AND KAHLER FORM ON $\mathbb{C} P^{2}$}

Here we follow $[7,14]$ and obtain the explicit form of the metric and of the Kahler form on $\mathbb{C} P^{2}$. We begin by defining $S^{5}$ as a sphere that is embedded in $\mathbb{C}^{3}$

$$
r^{2}=\left|Z^{0}\right|^{2}+\left|Z^{1}\right|^{2}+\left|Z^{2}\right|^{2}
$$

with the ambient flat space metric

$$
d s^{2}=\left|d Z^{0}\right|^{2}+\left|d Z^{1}\right|^{2}+\left|d Z^{2}\right|^{2} .
$$

We define inhomogeneous coordinates

$$
\zeta^{1}=\frac{Z^{1}}{Z^{0}}, \quad \zeta^{2}=\frac{Z^{2}}{Z^{0}},
$$

and put

$$
Z^{0}=\rho e^{i y}
$$

where

$$
\rho^{2}=\frac{r^{2}}{1+\sum_{a=1,2}\left|\zeta^{a}\right|^{2}}
$$

and

$$
y \sim y+2 \pi .
$$

We then get the metric on $S^{5}$ as

$$
d s^{2}=r^{2}\left((d y+V)^{2}+\frac{d \zeta^{a} d \bar{\zeta}^{a}}{1+\sum_{a}\left|\zeta^{a}\right|^{2}}-\frac{\zeta^{a} \bar{\zeta}^{b} d \bar{\zeta}^{a} d \zeta^{b}}{\left(1+\sum_{a}\left|\zeta^{a}\right|^{2}\right)^{2}}\right)
$$

where

$$
V=\frac{i}{2\left(1+\sum_{a}\left|\zeta^{a}\right|^{2}\right)}\left(\zeta^{a} d \bar{\zeta}^{a}-\bar{\zeta}^{a} d \zeta^{a}\right)
$$

If we parametrize

$$
\zeta^{1}=f(\chi, \psi) \cos \frac{\theta}{2} e^{\frac{i \varphi}{2}}, \quad \zeta^{2}=f(\chi, \psi) \sin \frac{\theta}{2} e^{-\frac{i \varphi}{2},}
$$

where

$$
f(\chi, \psi)=\tan \chi e^{\frac{i \psi}{2}}
$$

then we get

$$
d s^{2}=r^{2}(d y+V)^{2}+d s_{\mathbb{C P}^{2}}^{2}
$$

where

$$
\begin{aligned}
V & =\frac{1}{2} \sin ^{2} \chi \sigma_{3}, \\
d s_{\mathbb{C P}}^{2} & =r^{2}\left(d \chi^{2}+\frac{1}{4} \sin ^{2} \chi\left(\sigma_{1}^{2}+\sigma_{2}^{2}+\cos ^{2} \chi \sigma_{3}^{2}\right)\right),
\end{aligned}
$$


and

$$
\begin{aligned}
& \sigma_{1}=\sin \theta \cos \psi d \varphi-\sin \psi d \theta, \\
& \sigma_{2}=\sin \theta \sin \psi d \varphi+\cos \psi d \theta, \\
& \sigma_{3}=d \psi+\cos \theta d \varphi,
\end{aligned}
$$

for which we find that

$$
d \sigma_{3}=\sigma_{1} \wedge \sigma_{2}
$$

and cyclically related relations. We define $\tan \chi \geq 0$ so that $\chi \in[0, \pi / 2]$ and we make the identification

$$
\psi \sim \psi+4 \pi .
$$

We define the vielbein

$$
\begin{aligned}
e^{4} & =r d \chi, \quad e^{1}=\frac{r}{2} \sin \chi \sigma_{1}, \\
e^{2} & =\frac{r}{2} \cos \chi \sigma_{2}, \quad e^{3}=\frac{r}{2} \sin \chi \cos \chi \sigma_{3} .
\end{aligned}
$$

We then find that

$$
F=d V=\frac{2}{r^{2}} J
$$

where

$$
J=e^{4} \wedge e^{3}+e^{1} \wedge e^{2}
$$

is the Kahler form.

\section{APPENDIX E: THE VIELBEIN COMPONENTS} IN LIGHT-CONE COORDINATES

In light-cone coordinates on $\mathbb{R} \times S^{5}$, the vielbein has the components

$$
\left(\begin{array}{ccc}
e^{\hat{+}}+ & e^{\hat{+}}-e^{\hat{+}_{i}} \\
e^{\hat{\underline{-}}}+ & e^{\hat{-}}-e^{\hat{-}}{ }_{i} \\
e^{\hat{\hat{i}}} & e^{\hat{\hat{i}}} & e^{\hat{\hat{i}}}{ }_{i}
\end{array}\right)=\left(\begin{array}{ccc}
1 & 0 & \frac{r}{\sqrt{2}} \kappa_{i} \\
0 & 1 & -\frac{r}{\sqrt{2}} \kappa_{i} \\
0 & 0 & E^{\hat{i}}{ }_{i}
\end{array}\right)
$$

and its inverse is

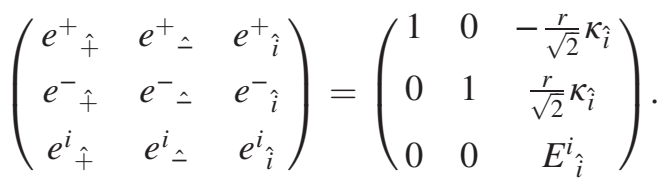

The metric is

$$
d s^{2}=-2 e^{\hat{+}} e^{\hat{\imath}}+e^{\hat{i}} e^{\hat{i}}
$$

and $E^{\hat{i}}$ denotes the vielbein on $\mathbb{C} P^{2}$. Since $\kappa_{i}$ is a Killing vector, we have the important identity

$$
\kappa^{i} w_{i j}=0
$$

where $w_{i j}$ is the Kahler form. Here $\kappa$ was denoted as $V$ and $w=d \kappa$ was denoted as $J$ in Appendix D.
[1] K. Hosomichi, R. K. Seong, and S. Terashima, Supersymmetric gauge theories on the five-sphere, Nucl. Phys. B865, 376 (2012).

[2] H. C. Kim and S. Kim, M5-branes from gauge theories on the 5-sphere, J. High Energy Phys. 05 (2013) 144.

[3] N. Lambert and T. Orchard, Null reductions of the M5-brane, J. High Energy Phys. 12 (2020) 037.

[4] N. Lambert and C. Papageorgakis, Non-Abelian $(2,0)$ tensor multiplets and 3-algebras, J. High Energy Phys. 08 (2010) 083.

[5] A. Gustavsson, A non-Abelian M5 brane Lagrangian in a supergravity background, J. High Energy Phys. 10 (2020) 001.

[6] A. Gustavsson, The non-Abelian tensor multiplet, J. High Energy Phys. 07 (2018) 084.

[7] H. C. Kim and K. Lee, Supersymmetric M5 brane theories on R x CP2, J. High Energy Phys. 07 (2013) 072.

[8] N. Lambert, A. Lipstein, and P. Richmond, Non-Lorentzian M5-brane theories from holography, J. High Energy Phys. 08 (2019) 060.
[9] N. Lambert, C. Papageorgakis, and M. Schmidt-Sommerfeld, M5-branes, D4-branes and quantum 5D super-YangMills, J. High Energy Phys. 01 (2011) 083.

[10] M. R. Douglas, On $D=5$ super Yang-Mills theory and $(2,0)$ theory, J. High Energy Phys. 02 (2011) 011.

[11] H. Linander and F. Ohlsson, $(2,0)$ theory on circle fibrations, J. High Energy Phys. 01 (2012) 159.

[12] J. Källén, J. Qiu, and M. Zabzine, The perturbative partition function of supersymmetric 5D Yang-Mills theory with matter on the five-sphere, J. High Energy Phys. 08 (2012) 157.

[13] N. Lambert and P. Richmond, (2,0) supersymmetry and the light-cone description of M5-branes, J. High Energy Phys. 02 (2012) 013.

[14] G. W. Gibbons and C. N. Pope, $\mathrm{CP}^{2}$ as a gravitational instanton, Commun. Math. Phys. 61, 239 (1978). 